\title{
Espectros de respuesta elásticos de desplazamiento para la ciudad de Armenia, apropiados para el diseño sísmico basado directamente en desplazamientos (dbdd)
}

\section{Elastic displacement response spectra suitable for applying direct displacement-based design (ddbd) in Armenia (Colombia)}

\author{
Juan Carlos Aldana Carrillo*, Andrés Rodríguez Mejia*, Hugo Monsalve Jaramillo*
}

Recibido: Junio 23 de 2009

Aceptado: Octubre 28 de 2009

Correspondencia: programa de Ingenieria, Universidad del Quindío, Avenida Bolivarcalle 12 norte Armenia Quindio. Email: hugom@uniquindio.edu.co

\begin{abstract}
RESUMEN
El diseño sísmico de estructuras ha ido evolucionando y tomando nuevos caminos, los cuales han conllevado a cambios radicales en los enfoques tradicionales de diseño. Es así, como las nuevas tendencias apuntan hacia el control de las deformaciones que experimentan las estructuras ante las cargas sísmicas, ya que es en éstas donde se puede determinar el daño estructural y no estructural. El control de las deformaciones se ha estudiado y presentado en lo que se conoce hoy en día como el diseño sísmico basado en desempeño, que ha ocasionado un cambio en la filosofía tradicional de diseño basado en fuerzas y a su vez, ha exigido cambios en las cargas sísmicas representadas en los espectros de respuesta de cualquier naturaleza. Este estudio se enfoca en lo que concierne a la presentación de espectros de respuesta elásticos de desplazamientos, que puedan ser utilizados en el diseño sísmico basado directamente en desplazamientos propuesto por Kowalsky y Priestley (2000), donde el desplazamiento es el parámetro de entrada para la aplicación de esta metodología. Por ello, se utilizó una metodología compuesta de dos partes: a) la sismológica representada en el ajuste de los parámetros libres que controlan la forma de los espectros de amplitudes de Fourier teóricos, para ser utilizados en la simulación de señales sísmicas utilizando técnicas estocásticas y b) la obtención de espectros de respuesta para los depósitos de suelo de la ciudad de Armenia. Es así como se obtuvieron 24 señales sintéticas para las parejas M-d definidos en Ramos (2006), acordes con la amenaza sísmica regional, utilizando el programa de simulación estocástica de Boore (2000) SMSIM, el cual esta basado en el modelo sismológico del espectro radiado para la simulación de movimiento fuerte del terreno. Dado que las señales generadas utilizando esta metodología no tienen en cuenta efectos locales, como lo es la amplificación de las ondas sísmicas en depósitos de suelo específicos, hubo la necesidad de filtrar Estas señales sintéticas a través de los depósitos de suelo de la ciudad de Armenia (Ingeominas, 1999), para así obtener señales que permitieran obtener espectros de respuesta de desplazamiento acordes con la normatividad que rige el diseño sísmico en la ciudad de Armenia (Decreto 079, 1999). Se generaron entonces espectros de respuesta de desplazamiento para tres zonas, al igual que los espectros de diseño para cada una de ellas, definidos para 6 niveles de amortiguamiento, apropiados para ser utilizados para el diseño sísmico basado directamente en desplazamientos en la ciudad de Armenia.

Palabras claves: Diseño sísmico, desempeño, espectros de respuesta, EAF, simulación estocástica, movimiento fuerte, efectos locales, desplazamiento.
\end{abstract}

\section{ABSTRACT}

The seismic design of structures has been evolving and taking new ways; as a consequence, new tendencies are aiming at controlling the deformation that structures experience when they undergo seismic loads, since it is with deformations where structural and non-structural damage can be determined. Control of deformations has been studied and presented in what is known today as Performance-Based Seismic Design, which has caused a change in the traditional design philosophy that is

*Centro de Estudios e Investigaciones de la Facultad de Ingeniería-CEIFI, Grupo Quimbaya, U. Quindío 
forced-based, and in turn, it has demanded changes in the seismic loads represented in the response spectra of any nature. This work focuses on obtaining elastic displacements response spectra that can be used in the displacement-based seismic design proposed for Kowalsky and Priestley (2000), where the displacement is the input parameter for the application of this methodology. For this purpose, a methodology consisting of two parts was used: a) seismological; represented in the adjustment of the free parameters that control the form of the Fourier amplitude spectra (FAS) to be used in the simulation of strong motion using stochastic methods and b) the obtaining of response spectra for soil deposits of Armenia city. As a consequence, 24 synthetic signals were obtained for the $M-d$ couple, consistent with the regional seismic hazard, using Boore's (2000) software for stochastic simulation SMSIM. This software is based on the seismological model of the radiated spectrum for the simulation of ground strong motion. Since the generated signals using this methodology do not take into account site effects; namely, the amplification of the seismic waves in specific soil deposits, it was necessary to filter these synthetic signals through the soil deposits in city of Armenia (Ingeominas, 1999). Consequently, it was possible to generate signals that allowed obtaining displacement response spectra consistent with the seismic code that regulates the seismic design in Armenia (Decree 079, 1999). Both displacement response spectra and displacement design spectra for three zones were generated (zones $A, B$ and C); all of them were defined for six levels of damping, appropriate to be used for displacement-based seismic design in Armenia.

Key works: seismic design, performance, response spectra, FAS, stochastic, simulation, ground motion, site effects, and displacement.

\section{INTRODUCCIÓN}

D ía a día el diseño sismorresistente avanza de la mano con la tecnología, principalmente con el apoyo de ésta en la disponibilidad de equipos básicos, que van desde un computador hasta equipos más sofisticados como acelerógrafos digitales. Todo esto, sumado a la dedicación de grupos de investigación centrados en derribar conceptos y filosofías de diseño clásicas, da como resultado el crecimiento y desarrollo del diseño conceptual. Conjugados estos principios, conlleva a que se estén reevaluando las metodologías tradicionales de diseño basado en fuerzas; y lo que sucede es que en los últimos tiempos, los investigadores se han dado cuenta de que el diseño sísmico debe de ir más allá y encontrar el verdadero significado del mismo. Ante una carga sísmica la estructura manifiesta deformaciones en todos sus elementos de resistencia, logrando incursionar en el rango inelástico y poder disipar gran parte de la energía mediante rotulaciones plásticas en las zonas confinadas. Básicamente, el objetivo de la ingeniería sísmica es la garantizar que sucedan dichos mecanismos. Desde esta perspectiva, el diseñador estructural debe darle más importancia a las deformaciones de la estructura y conocer ante diferentes eventos sísmicos, las deformaciones impuestas a la estructura y por consiguiente las demandas de ductilidad de los elementos. Conscientes de la necesidad de manejar el diseño sismorresistente desde el campo de las deformaciones, se han desarrollado metodologías para hacer de éstos el parámetro principal de diseño (SEAOC, 1995). Entre las metodologías desarrolladas se encuentra la de diseño basado directamente en desplazamiento (Kowalsky, Priestley, 2000), aunque ésta hace parte de un grupo de metodologías todas enfocadas a cumplir con el diseño basado en desempeño (FEMA, 1997). Un análisis detallado de estas metodologías se presenta en Sullivan (2002). Dadas éstas nuevas tendencias a nivel mundial, Colombia y para este caso la ciudad de Armenia, no deben de ser ajenas a esta gran realidad. Por ello se han realizado estudios encaminados para dar a conocer éstas tendencias; pero específicamente en el diseño basado directamente en desplazamientos se conoce el trabajo de Restrepo (2003), en donde se concluyó que para la aplicación del DBBD, no había los espectros de desplazamientos adecuados para ser aplicados en la metodología, por lo cual se debían encaminar esfuerzos para determinar dichos espectros, teniendo en cuenta las limitaciones y formas de su obtención (Bommer, Elnashai, 1999). Utilizando una metodología basada en la obtención de señales sintéticas a partir de la simulación estocástica de movimiento fuerte (Boore, 2003), se generaron señales para las parejas M-d (magnitud-distancia) dadas en Ramos (2006), las cuáles se filtraron por los depósitos de suelo dados en la microzonificación indicativa de la ciudad de Armenia (Ingeominas, 1999), para obtener así señales de aceleración en suelo con las cuales se generaron los espectros de desplazamiento elásticos para la ciudad de Armenia.

\section{CARACTERIZACIÓN DE LOS ESPECTROS DE AMPLITUDES DE FOURIER (EAF)}

Los sismólogos han encaminado esfuerzos para describir mediante modelos físicos de dislocación (e.g. Haskell, 1966) la fenomenología de la energía radiada en una fuente y cómo representarla mediante formulaciones matemáticas y físicas, llegando a modelos cómo los de Brune (1970) que describe la radiación de amplitudes de la fuente y Aki (1967) quien propuso el modelo de fuente, donde las amplitudes a altas 
frecuencias decaen de acuerdo la relación.

\section{Espectro radiado: Modelo de fuente puntual.}

Para distancias mayores a 5 veces el radio de la falla circular equivalente (Gallego y Ordaz, 1999), el espectro de amplitudes de fourier de aceleración utilizado para describir la radiación de la alta frecuencia de las ondas de cortante (S) se da en la ecuación 1 , la cual es una función del momento sísmico y la distancia.

$$
A(M o, R, f)=E(M o, f) D(R, f) P(f) I(f)
$$

Esta representación que se da en Boore (1983), deja ver claramente los términos que afectan la forma del espectro de amplitudes de aceleración, donde $E(M o, f)$ es el espectro de fuente, $D(R, f)$ es una función que modela la atenuación anelástica y geométrica como una función de la distancia Hipocentral y la frecuencia, $P(f)$ es un filtro que reduce rápidamente las amplitudes a alta frecuencia, el cual pude ser basado en el modelo $f_{\max }$ (Hanks, 1982) o el modelo Kappa (Anderson and Hough, 1984). El término $I(f)$ es un filtro usado para la forma del espectro correspondiente a un movimiento del terreno en particular (Aceleración, Velocidad o Desplazamiento). La forma del espectro antes descrito se puede observar en la figura 1 , en donde se ve el decaimiento de las amplitudes a altas frecuencias, tal como se observa en los espectros reales; en la ecuación 1 los términos que acompañan al espectro de fuente son los que permiten que se observe dicho decaimiento, ya que el espectro de fuente describe amplitudes constantes para altas frecuencias.

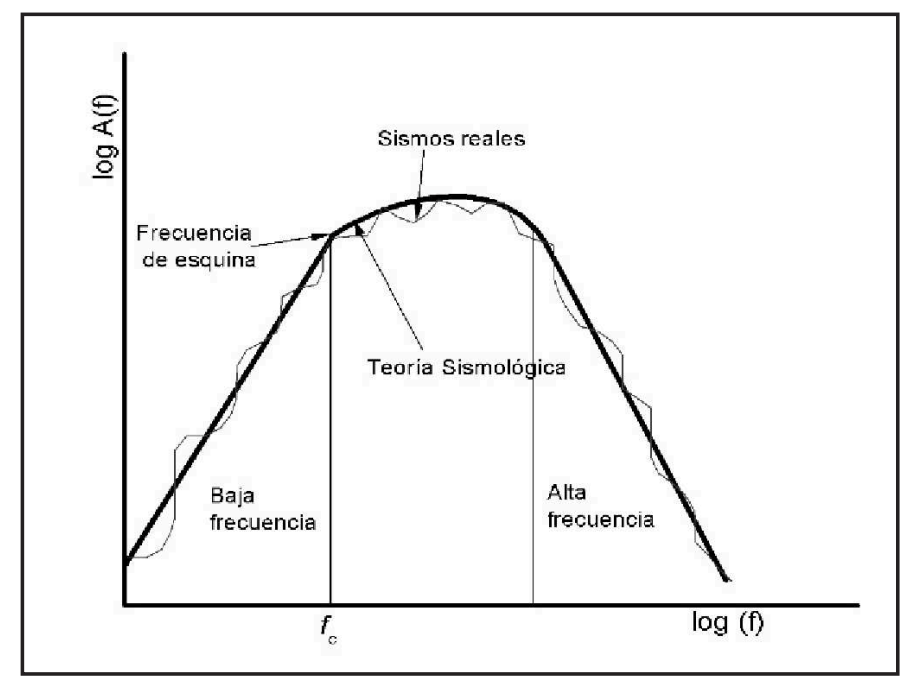

Figura 1: Forma típica del Espectro de Amplitudes de Fourier, descrito en la ecuación 1

\section{Espectro radiado: Modelo de fuente finita.}

Se ha observado que a distancias focales muy pequeñas, comparables con el tamaño de la ruptura, el modelo de fuente puntual falla. Por lo cual para contar con un modelo útil en las cercanías de la fuente, Singh et al. (1989) desarrollaron un modelo sismológico que tiene en cuenta el tamaño finito de la zona de ruptura. El modelo contempla una falla circular de radio ro $\quad$ (Ec. 2) que rompe con intensidad uniforme a lo largo del área. El punto de observación $P$ esta situado justo arriba del foco que se localiza a una distancia R0 sobre el hipocentro.

$$
r_{0}=2.34 \frac{\beta}{\omega_{C}}
$$

donde $\mathrm{r} 0$ es el radio equivalente de una falla circular en $\mathrm{km}$, $\omega c=2 \pi f c$ y $\beta$ la velocidad de la onda $\mathrm{S}$.

En la ecuación No. 3 se pude ver la representación propuesta en el trabajo de Gallego (2000), la cual fue utilizada en este estudio para la calibración de los parámetros libres $R_{\theta \phi}, Q_{o}, Q_{l}, \kappa_{l}, \varepsilon$. Donde $R_{\theta \phi}$ es el patrón de radiación, $Q_{o}$ el índice de calidad de atenuación a $1 \mathrm{~Hz}, Q_{l}$ es el índice de calidad de atenuación para efectos locales, $\varepsilon$ es el coeficiente para la atenuación en función de las frecuencias.

$$
\begin{aligned}
& (A(f))^{2}=4 R_{\vartheta \phi}{ }^{2} \cdot C^{2} \cdot\left(M_{0} f_{C}^{2}\right)^{2} \cdot \frac{e^{\left(-2 \pi\left(k_{1}+\frac{R}{Q_{1}}\right) f\right)}}{\left(r_{0}\right)^{2}} \cdot\left[E 1\left(\alpha \cdot R_{0}\right)-E l\left[\alpha \cdot \sqrt{\left(r_{0}^{2}+R_{0}^{2}\right)}\right]\right] \\
& C=\frac{1}{4 \pi \rho \beta^{3}}
\end{aligned}
$$

E1( ) es la integral exponencial (Gautschi y Cahill, 1965) y $\alpha=2 \pi / \beta Q 0, \rho$ es la densidad y $\beta$ la velocidad de la onda $S, f_{c}$ es la frecuencia de esquina, esta integral tiene solución numérica, la solución matemática fue resuelta por Monsalve (2005) (comunicación personal).

El modelo de fuente finita parte de la suposición de que el observador se encuentra a una distancia cercana. Como ya se vio anteriormente, el radio de la falla $r_{0}$ equivalente crecerá a medida que aumente la magnitud; si esto sucede, y teniendo en cuenta que las ondas sufren todos los fenómenos de atenuación descritos anteriormente, la contribución de ondas que vienen del cada vez más lejano perímetro sufrirá los procesos exponenciales de atenuación, haciendo que la aceleración no crezca de forma indefinida. Este comportamiento es el que provoca la saturación de la aceleración para magnitudes muy grandes y distancias cercanas al epicentro.

Las ecuaciones utilizadas para calibrar los parámetros libres son las que se presentan a continuación (Ec. 4 y 5 ):

Si $R<R x$, donde R es la distancia epicentral:

$(A(f))^{2}=4 R \theta \phi^{2} \cdot C^{2} \cdot\left(M_{0} f_{C}^{2}\right)^{2} \cdot \frac{e^{\left(-2 \pi\left(k_{1}+\frac{R}{Q}\right) f\right)}}{\left(r_{0}\right)^{2}} \cdot\left[E \mathrm{l}\left(\alpha \cdot R_{b}\right)-E 1\left[\alpha \cdot \sqrt{\left(r_{0}^{2}+R_{b}^{2}\right)}\right]\right]$ 
Si R $>$ Rx

$\left.A(f, R)=\frac{2}{\sqrt{2}} \cdot R \theta \phi \cdot C \cdot \frac{M_{0} f^{2}}{1+\left(\frac{f}{f_{C}}\right)^{2}} \cdot \frac{e^{\left(\frac{-\pi f R}{\beta Q_{0} f^{\varepsilon}}\right)}}{\sqrt{R \cdot R x}} \cdot e^{\left(-\pi\left(k_{1}+\frac{R}{Q_{1}}\right) f\right.}\right)$

Gallego y Ordaz (1999) encontraron que una distancia Rx para la cual existe un buen acoplamiento es 5 veces el radio de la falla equivalente de ruptura (ver ec. 4 y 5 ), donde el acoplamiento se refiere a la condición donde las dos ecuaciones mostradas convergen a un mismo valor de aceleración. También hay que tener en cuenta que si el movimiento de suelo va a estar expresado en $\mathrm{cm} / \mathrm{s}$ y $\rho$, $\beta$, y RO están en las unidades de $\mathrm{gm} / \mathrm{cm} 3, \mathrm{~km} / \mathrm{s}$ y $\mathrm{km}$, respectivamente, entonces las ecuaciones anteriores deben multiplicarse por el factor 10-20, obtenido compatibilizando unidades.

En las ecuaciones anteriores Rx es la distancia que diferencia el campo cercano del lejano, esta dada como 5 veces el radio de la falla equivalente (Gallego y Ordaz, 1999), $f$ es frecuencia en $\mathrm{Hz}, \mathrm{R}$ es la distancia hipocentral de la estación con respecto al evento en $\mathrm{km}, \mathrm{R} \theta \phi$ es el patrón de radiación que será motivo de calibración en este trabajo, $\rho$ es la densidad de la roca en la región focal, $2.8 \mathrm{~g} / \mathrm{cm} 3$ para zona "Activa" (se refiere a la corteza) y $3.3 \mathrm{~g} / \mathrm{cm} 3$ para zona "Subducción" y $\beta$ es la velocidad de onda cortante en la región focal, $3.5 \mathrm{~km} / \mathrm{s}$ para zona "Activa" y $4.68 \mathrm{~km} / \mathrm{s}$ para zona "Subducción" (Ingeominas, 1993). El momento sísmico M0 es definido por Kanamori (1977), en dina-cm. Debido a que los sismo registrados por la red de acelerógrafos de Colombia se reportan con magnitud $\mathrm{Ml}$, se utilizaron las correlaciones de magnitud $\mathrm{Ml}$ y magnitudes $\mathrm{mb}$ reportadas por USGS derivadas de Ojeda y Martínez (1998), algunos de los sismos registrados por la red a acelerógrafos tienen reporte de magnitud $\mathrm{Mw}$ en la pagina Web de NEIC; los parámetros $\mathrm{Q} 0, \varepsilon, \mathrm{Ql}, \mathrm{kl}$ ya fueron descritos anteriormente $y$ serán motivo de calibración junto con la caída de esfuerzos $(\Delta \sigma)$.

\section{SIMULACIÓN ESTOCÁSTICA DE MOVIMIENTO FUERTE}

En la simulación estocástica de movimiento fuerte, se necesitan conocer los parámetros libres de los espectros de amplitudes de Fourier (EAF) que representan la sismicidad regional, para así obtener las señales sintéticas para un sismo en particular. Estos parámetros están contenidos en el espectro de amplitudes de Fourier (EAF) obtenido de modelos físicos, en el cual se describen el proceso de ruptura en la fuente (radiación de la energía sísmica), el viaje de las ondas a través del medio y la disminución de la alta frecuencia.

\section{El proceso de simulación de señales.}

La esencia del método se puede observar en la figuras 2 y 3 , donde la energía radiada descrita por los espectros de amplitudes de Fourier (cuadro izquierdo de la figura), es asumida que se distribuye aleatoriamente sobre una duración igual a la inversa de la frecuencia de esquina más baja (fo) (Boore, 2003).
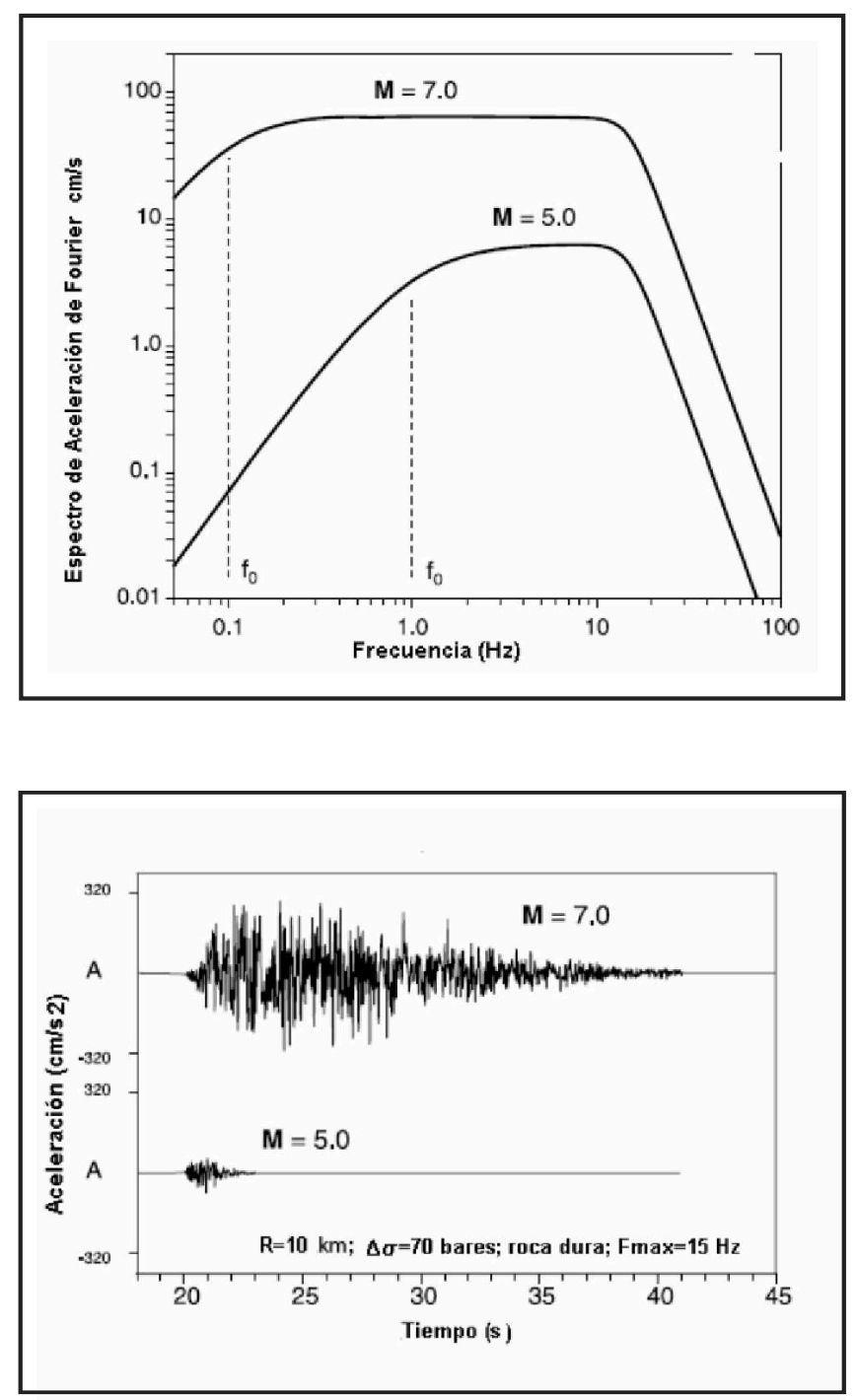

Figura 2: La energía radiada descrita por el espectro en la figura izquierda, es asumida que se distribuye aleatoriamente sobre una duración igual a la inversa de la frecuencia de esquina más baja (fO). Cada serie de tiempo en la figura derecha, es una realización del proceso aleatorio para el espectro real mostrado. Cuando es trazado en escala logarítmica, los niveles de la parte de baja frecuencia de los espectros son directamente proporcionales al logaritmo del momento sísmico y así a la magnitud del momento (Modificado de Boore, 2003). 

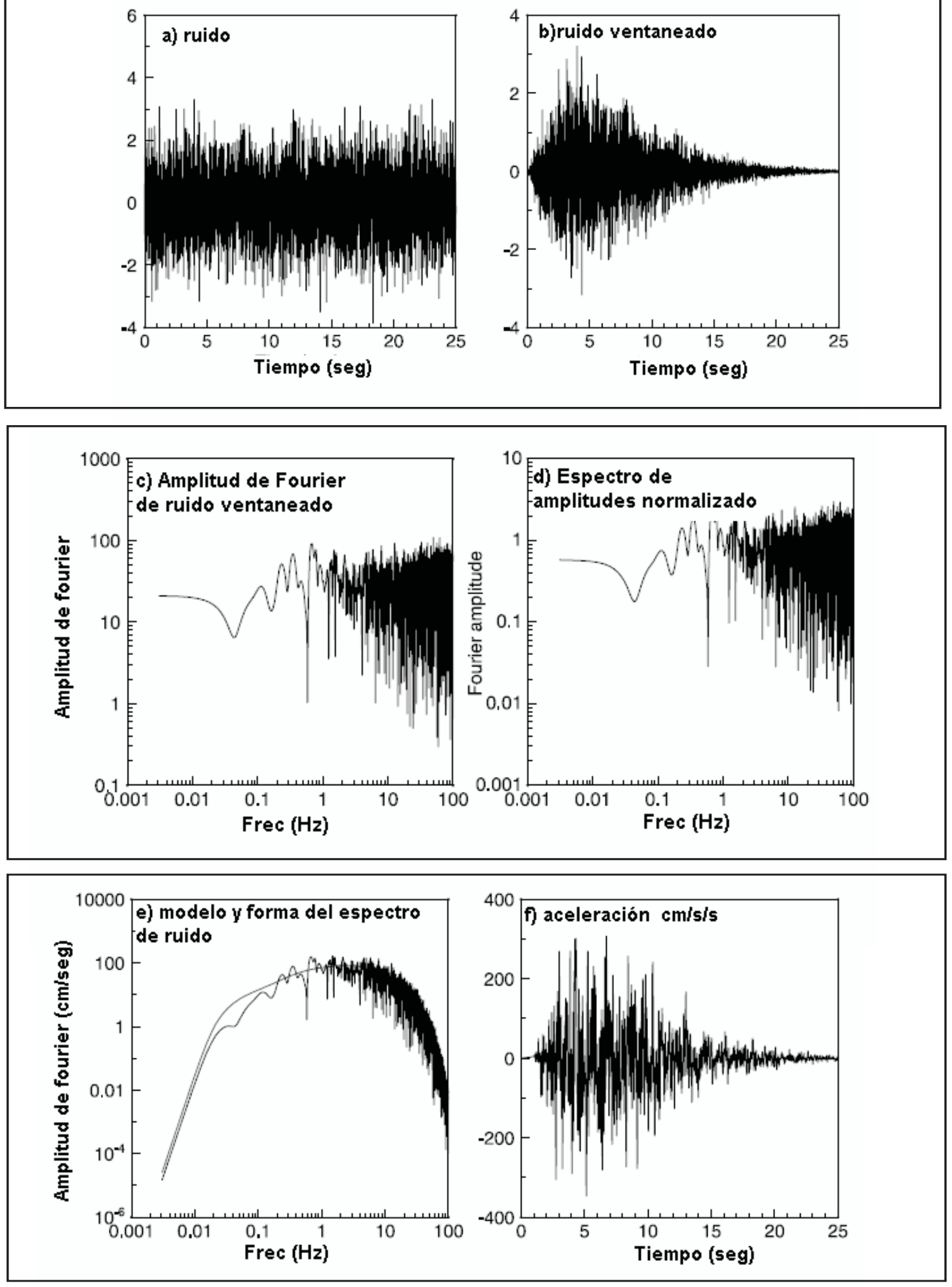

Figura 3. En la figura se observan los diferentes pasos, en el dominio del tiempo y la frecuencia, para simular movimientos de suelo usando el método estocástico (Modificado de Boore, 2003). 


\section{Función de duración del movimiento fuerte}

La duración del movimiento Tgm depende de la distancia de propagación y del tiempo que dura el proceso de ruptura. Aunque los espectros de amplitudes de Fourier no tienen en cuenta directamente la duración del movimiento, el método de simulación de movimiento fuerte permite mediante una simple función involucrar el tiempo.

Para la determinación de la duración del movimiento fuerte, consistente con la teoría de procesos aleatorios, se debe calcular la duración requerida que ajuste las relaciones observadas entre las amplitudes del dominio del tiempo y el dominio de la frecuencia, esto se denomina como la duración rms (Atkinson, 1993a). Del teorema de Parseval:

$$
v_{m s}=\left[2 / T \int_{0}^{\infty} V^{2}(f) d f\right]^{1 / 2}
$$

Donde $v_{r m s}$ es la raíz de la velocidad cuadrática media, $\mathrm{V}(f)$ es el valor absoluto del espectro de Fourier de velocidad, y $f$ es la frecuencia $(\mathrm{Hz})$, T es el tiempo a determinar.

De la teoría de procesos aleatorios,

$$
V p=P v_{r m s},
$$

Donde $V p$ es la velocidad pico del terreno y $P$ es el factor pico, dado por (Cartwright y Longuet-Higgins, 1956)

$$
P=(2 \ln (N))^{1 / 2}+0.5772 /(2 \ln (N))^{1 / 2}
$$

Donde $\mathrm{N}$ es el número de extremos, tomado como

$$
N=2 F T
$$

Donde $F$ es la frecuencia predominante y $T$ es el tiempoa determinar.

Una vez se obtiene la duración total ésta se descompone en dos tiempos, el primero es la duración de la ruptura, la cual se relaciona como la inversa de la frecuencia de esquina (depende del modelo de fuente que se utilice); el segundo tiempo corresponde a la trayectoria, que es posible representarla mediante regresiones lineales en diferentes rangos de distancia, obtenidas de registros observados tal y como puede apreciarse en la función utilizada por Atkiston y Boore (1995).

\section{METODOLOGÍA Y RESULTADOS}

PROCESAMIENTO DE DATOS. Se procesaron 254 señales de componentes digitales horizontales correspondientes a 54 eventos sísmicos, registrados en la Red Nacional de Acelerógrafos de Colombia (RNAC) del INGEOMINAS, en el período junio de 1993 a mayo de 2005. La figura 4 muestra la localización epicentral de los eventos. A las componentes horizontales de cada estación se le hizo corrección instrumental teniendo en cuenta para ello la sensitividad, frecuencia natural de oscilación y amortiguamiento de los acelerómetros, se corrigió por línea base para evitar la desviación de los acelerogramas respecto al centro (offset) y las señales fueron cortadas antes del arribo de la onda $\mathrm{P}$, con el fin de aplicar un filtro Butterworth de 0.2 a $10 \mathrm{~Hz}$. Una vez obtenido el espectro de amplitudes de fourier del arribo de la onda S de cada componente horizontal, se calculó la media cuadrática de la siguiente manera:

$$
E A F_{p r o m}=\sqrt{\frac{E A F_{E W}^{2}+E A F_{N S}^{2}}{2}}
$$

ANÁLISIS DE ESPECTRO DE FUENTE. El procedimiento de análisis consiste en ajustar los parámetros libres del espectro de amplitudes de Fourier tal como es descrito en Gallego (2000). En este trabajo se utilizó el algoritmo de LevenbergMarquardt como método de minimización no lineal para el ajuste de los parámetros libres del EAF. Para realizar el análisis se separaron los sismos de Colombia en dos tipos representativos de la tectónica colombiana: 1) sismos registrados en las zonas de subducción o zona de Benioff , bajo la placa continental que se denominará "Subducción"; y 2) movimientos sísmicos de fallas corticales en el territorio colombiano, que se denominará "activa" . Adicionalmente se realizó un análisis para el tipo "Activa" donde se separaron en tres zonas representativas de sismicidad: Oriental, Central, Occidental, las cuales se encuentran separadas como se muestra en la figura 5.

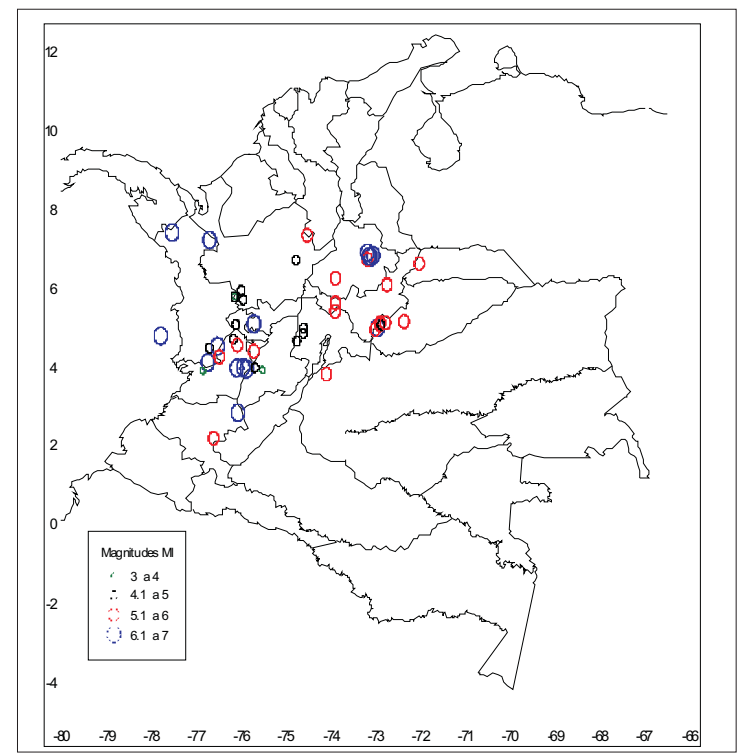

Figura 4. Se muestran los epicentros de sismos de 1993 a 2005 , utilizados en este estudio 
Ya asignados los sismos registrados por la Red Nacional de Acelerógrafos a cada fuente sísmica (Activa y Subducción) se procedió a realizar el ajuste de los parámetros libres de los espectros teóricos de amplitudes de Fourier.

El procedimiento de regresión no lineal, utilizando el algoritmo del Levenberg-Marquardt, se realizó iterativamente con cada sismo y las señales que se registraron en cada estación; como se pudo observar, en algunos sismos solo se obtuvo uno o dos trazas digitales, lo cual ya es un problema debido a la poca información que se dispone. A pesar de esto se ajustan los parámetros libres enunciados previamente, teniendo en cuenta que en el EAF la amplitud es la variable dependiente y la frecuencia es la variable independiente. Se calculó en la iteración una caída de esfuerzos $(\Delta \sigma)$ por sismo, dejando que los parámetros de calidad $(\mathrm{Q} 0, \varepsilon, \mathrm{Ql}, \mathrm{kl})$ y el patrón de radiación $(\mathrm{R} \theta \phi)$ variaran respecto al sitio de la estación y su trayectoria.

ANÁLISIS DE RESULTADOS DE LA CALIBRACIÓN DEL EAF. AI realizar el análisis de minimización, con el algoritmo de Levenberg-Marquardt, se procedió a construir los espectros de fuente finita y fuente puntual para cada combinación de magnitud y distancia. La figura 6 muestra las formas típicas de este tipo de espectros. Ya que el espectro de fuente finita genera amplitud constante para las bajas frecuencias, en ese rango se usa el valor mínimo correspondiente al espectro de fuente puntual, Singh et al. (1989).
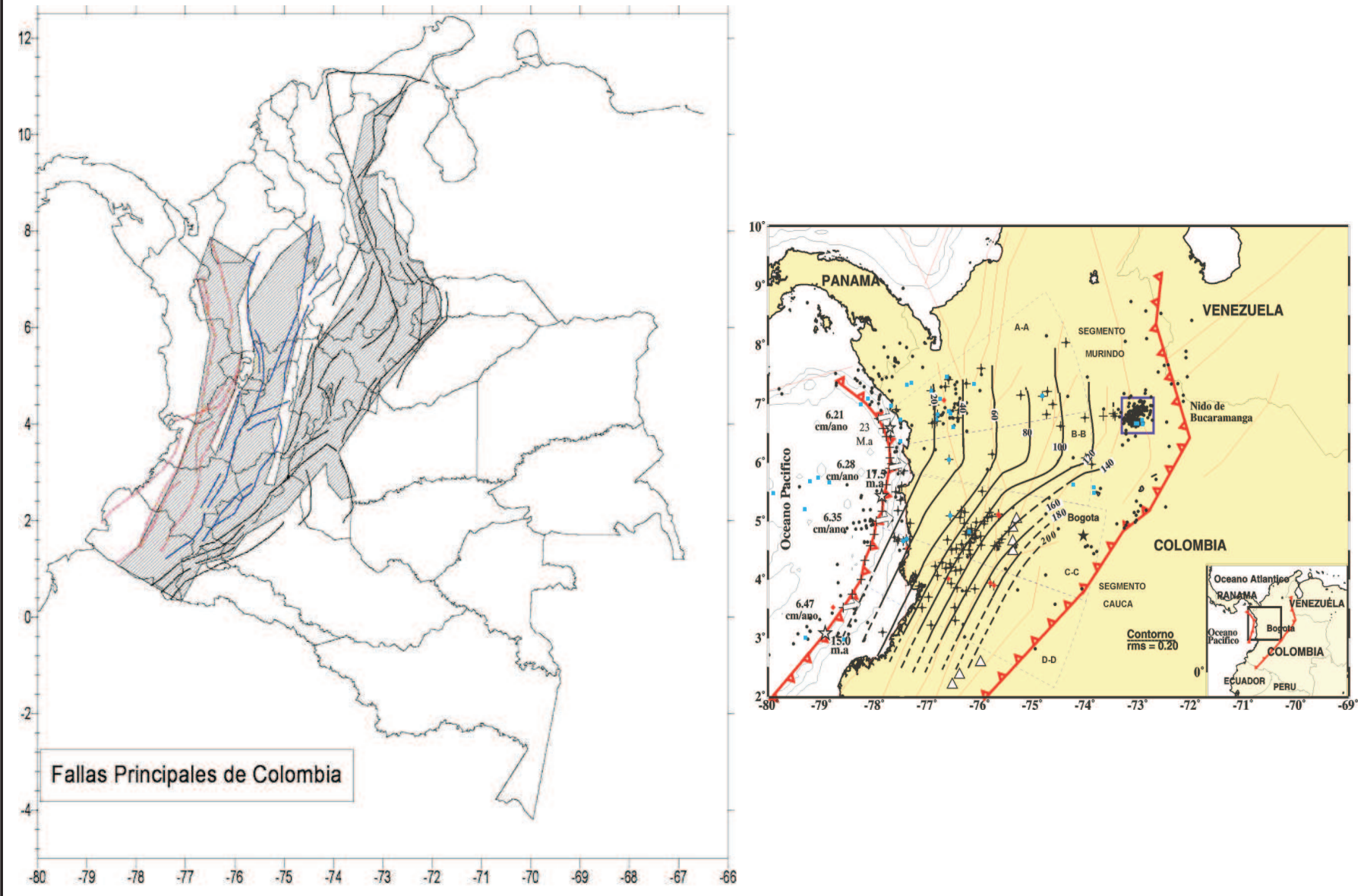

Figura 5. Izquierda: Distribución de las fuentes continentales que generan amenaza en el territorio colombiano; que corresponden a fallamientos corticales, denominado zona activa. derecha: isoprofundida-des de la subducción de la placa Nazca o zona de Benioff en el territorio que generan amenaza significativa. (Tomada de Monsalve H., 1998). 


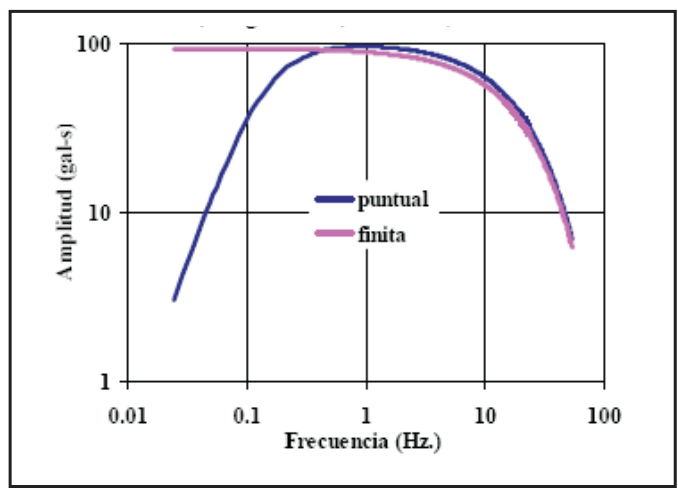

Figura 6. Modelos de fuente puntual y fuente finita para una combinación de magnitud y distancia.
Debido a que en la sismicidad registrada por la Red Nacional de Acelerógrafos, no se cuenta con registros de sismos correspondientes al campo cercano, esto conllevó a que sólo se trabajara con el EAF de campo lejano. Para validar la integral exponencial (Gautschi y Cahill, 1965) que fue resuelta analíticamente. Se realizó el análisis de regresión no lineal trabajo con una señal que tuviera las condiciones de campo cercano, esta señal fue buscada en los catálogos internacionales.

En la tabla 1 se muestran los resultados de la calibración de los parámetros para cada caso analizado.

Tabla 1: Parámetros calibrados para cada caso analizado

\begin{tabular}{|c|c|c|c|c|c|c|}
\hline ZONA & Q1 & $\varepsilon$ & Q0 & $\mathbf{R} \theta \boldsymbol{\varphi}$ & K1 & $\Delta \sigma$ (bares) \\
\hline Activa & 1754.23 & 1.37 & 231.59 & 0.60 & 0.0148 & 143.42 \\
\hline Occidental & 2164.89 & 1.33 & 379.66 & 0.61 & 0.0164 & 143.42 \\
\hline Central & 1980.63 & 1.23 & 177.66 & 0.60 & 0.0080 & 143.42 \\
\hline Oriental & 1437.01 & 1.34 & 192.83 & 0.60 & 0.0167 & 143.42 \\
\hline Subducción & 2447.74 & 1.44 & 255.70 & 0.59 & 0.0150 & 288.42 \\
\hline
\end{tabular}

\section{SIMULACIÓN DE SEÑALES}

\section{Parejas M-d.}

En el estudio de desagregación de la amenaza sísmica regional realizado por Ramos (2006), se propuso unas parejas magnitud-distancia para las diferentes fuentes que generan una amenaza representativa para la ciudad de Armenia; se optó por utilizar dichas parejas para generar las respectivas señales utilizando la metodología propuesta por Boore, (2003) adoptada en este estudio. En total se generaron 8 señales para las parejas aportadas por la falla Ibagué, 8 para Romeral y 8 para la zona de subducción. Estas señales representativas de la amenaza sísmica que tiene la ciudad de Armenia, se utilizarán para la generación de espectros de respuesta de desplazamiento para las zonas especificadas en el decreto 079 que reglamenta la microzonificación de Armenia.

En las tablas 2 a 4 se ven los valores de magnitud y distancia para los sistemas de fallas Romeral, Ibagué y zona de subducción, obtenidas en Ramos(2006).
Tabla 2. Ternas $M-d-\sigma$ para la falla Romeral

\begin{tabular}{|c|c|c|}
\hline $\begin{array}{c}\text { Magnitud } \\
(\mathrm{Mw})\end{array}$ & $\begin{array}{c}\text { Distancia (d) } \\
\mathrm{Km}\end{array}$ & $\begin{array}{c}\text { Desviación } \\
(\sigma)\end{array}$ \\
\hline 6.5 & 21.41 & 0.8 \\
\hline 6.5 & 18.4 & 0.6 \\
\hline 6.4 & 19.65 & 0.8 \\
\hline 6.4 & 16.58 & 0.6 \\
\hline 6.3 & 18.05 & 0.8 \\
\hline 6.3 & 15.48 & 0.6 \\
\hline 6.2 & 16.68 & 0.8 \\
\hline 6.2 & 14.26 & 0.6 \\
\hline
\end{tabular}


Tabla 3. Ternas $M-d-\sigma$ para la falla Ibagué

\begin{tabular}{|c|c|c|}
\hline $\begin{array}{c}\text { Magnitud } \\
(\mathrm{Mw})\end{array}$ & $\begin{array}{c}\text { Distancia }(\mathrm{d}) \\
\mathrm{Km}\end{array}$ & $\begin{array}{c}\text { Desviación } \\
\left(\begin{array}{c}\sigma\end{array}\right)\end{array}$ \\
\hline 6.8 & 65.48 & 0.8 \\
\hline 6.8 & 56.02 & 0.6 \\
\hline 6.7 & 59.17 & 0.8 \\
\hline 6.7 & 50.86 & 0.6 \\
\hline 6.6 & 54.03 & 0.8 \\
\hline 6.6 & 46.61 & 0.6 \\
\hline 6.5 & 49.56 & 0.8 \\
\hline 6.5 & 43.27 & 0.6 \\
\hline
\end{tabular}

Tabla 4. Ternas $M-d-\sigma$ p\&ra la Zona de Subducción.

\begin{tabular}{|c|c|c|}
\hline $\begin{array}{c}\text { Magnitud } \\
(\mathrm{Mw})\end{array}$ & $\begin{array}{c}\text { Distancia }(\mathrm{d}) \\
\mathrm{Km}\end{array}$ & $\begin{array}{c}\text { Desviación } \\
\left(\begin{array}{c}\sigma\end{array}\right)\end{array}$ \\
\hline 8.0 & 223.63 & 0.8 \\
\hline 8.0 & 190.91 & 0.6 \\
\hline 7.9 & 203.46 & 0.8 \\
\hline 7.9 & 173.53 & 0.6 \\
\hline 7.8 & 176.73 & 0.8 \\
\hline 7.8 & 152.15 & 0.6 \\
\hline 7.7 & 160.48 & 0.8 \\
\hline 7.7 & 138.41 & 0.6 \\
\hline
\end{tabular}

\section{Obtención función del tiempo}

Para realizar las simulaciones de movimiento fuerte $y$ obtener las señales deseadas, se utilizó el paquete de programas en Fortran, SMSIM desarrollado por Boore (2000). El programa SMSIM, utiliza una curva de tiempo - distancia representada mediante ecuaciones de líneas rectas, en las cuales se define la duración del movimiento fuerte de la señal a simular. Para la obtención de éstas curvas se determinó primero que todo la duración total del movimiento fuerte, es decir, el tiempo de ruptura mas el tiempo de propagación de la onda; luego se le restó el tiempo de ruptura, dado como $1 / f_{0}$ (Atkinston, Boore, 1995), para así obtener el tiempo de propagación de la onda. La función de tiempo obtenida en este estudio se puede observar en la figura 7. Para determinar la forma de la curva de tiempo a utilizar, se realizaron regresiones lineales en los tres tramos de diferente pendiente, ajustando la recta para cada tramo que mejor representara los puntos dispersos. Se tomó el modelo de tiempo con las ecuaciones de tres líneas en rangos de distancia de 0 a $100 \mathrm{~km}$, de 100 a $200 \mathrm{~km}$ y de 200 a $300 \mathrm{~km}$, debido a que la distribución de puntos así lo muestra, uniendo los puntos medios estimados en rangos de intervalo de distancia de $15 \mathrm{~km}$.

Para aplicar esta metodología, hubo la necesidad de restar el tiempo de ruptura dado en Atkinson and Boore (1995), puesto que de no ser así, los tiempos no dependerían sólo de la propagación y distancia, sino que estarían "escalados" por la magnitud del sismo, haciendo imposible el manejo estadístico para la generación de las rectas que describen la función del tiempo.

Las ecuaciones que se obtuvieron para cada tramo de recta son las siguientes: $\mathrm{De} 0 \mathrm{~s}$ a $10 \mathrm{~s}=0$

$$
\begin{array}{lr}
t=-1.16222+0.11622 d & \text { (tramo 1) } 0 \leq D<100 \mathrm{Km} \\
t=11.12-0.066 d & \text { (tramo 2) } 100 \leq D<200 \mathrm{Km} \\
t=-3.02667+0.06413 d & \text { (tramo 3) } 200<D \leq 300 \mathrm{Km}
\end{array}
$$

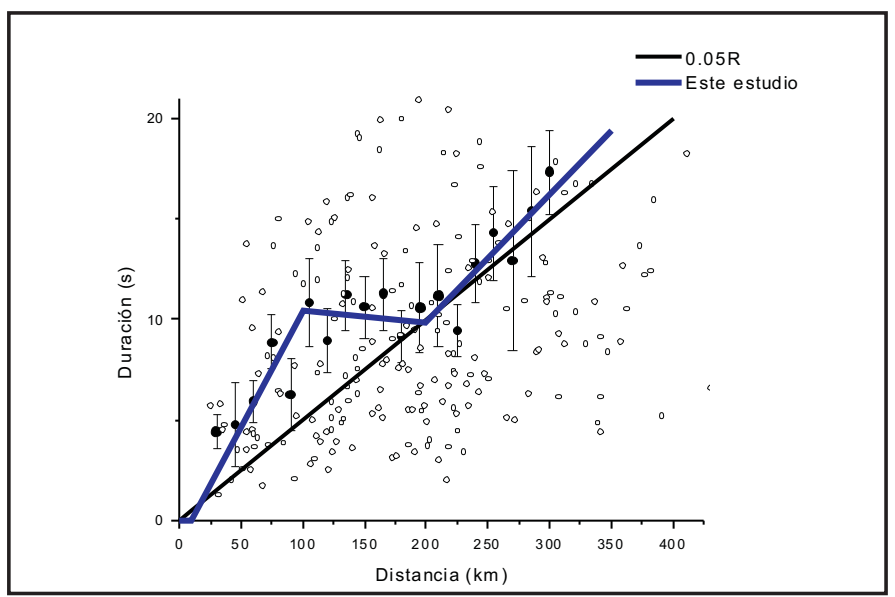

Figura 7. Función de tiempo utilizada para la simulación de movimiento fuerte. Los círculos sólidos son rangos de promedio en ventanas de $15 \mathrm{~km}$ (celdas) y las barras de error corresponden a la media más o menos una desviación estándar. La línea sólida (con tres segmentos) es la función usada para las simulaciones de este estudio. 


\section{Esparcimiento geométrico}

En la caracterización de los espectros de amplitudes de fourier, se tomo como modelo de esparcimiento geométrico las expresiones $1 / R$ y $\left(R^{*} R x\right)-1 / 2$ (Ordaz y Singh, 1992), en donde el primer término modela la atenuación geométrica para distancias en la cuales el movimiento ondulatorio es gobernado por las ondas de cuerpo, mientras que el segundo se refiere a la atenuación de las ondas sísmicas para distancias donde predominan las ondas superficiales. Este modelo se ha utilizado en estudios previos, tomando una distancia de $100 \mathrm{~km}$ como apropiada para la utilización de estas ecuaciones. En diversos estudios como los de Atkinston y Mereu (1992), se expone que estos modelos de atenuación han sido cuestionados, pues se ha encontrado en estudios de propagación de onda (Burguer et al. 1987) que las amplitudes espectrales decaen de una forma compleja para un modelo de corteza simple. Es así como se decidió aplicar este modelo de tres segmentos para la zona de Fallas activas y subducción, con el fin de obtener unos modelos de atenuación geométrica para el caso Colombiano.

\section{Esparcimiento geométrico: Zona Activa}

Para este estudio se obtuvieron las curvas de atenuación geométrica para la zona activa y de subducción. Las curvas se adecuaron a un modelo trilineal y se presentan en la gráfica 8 y 9:

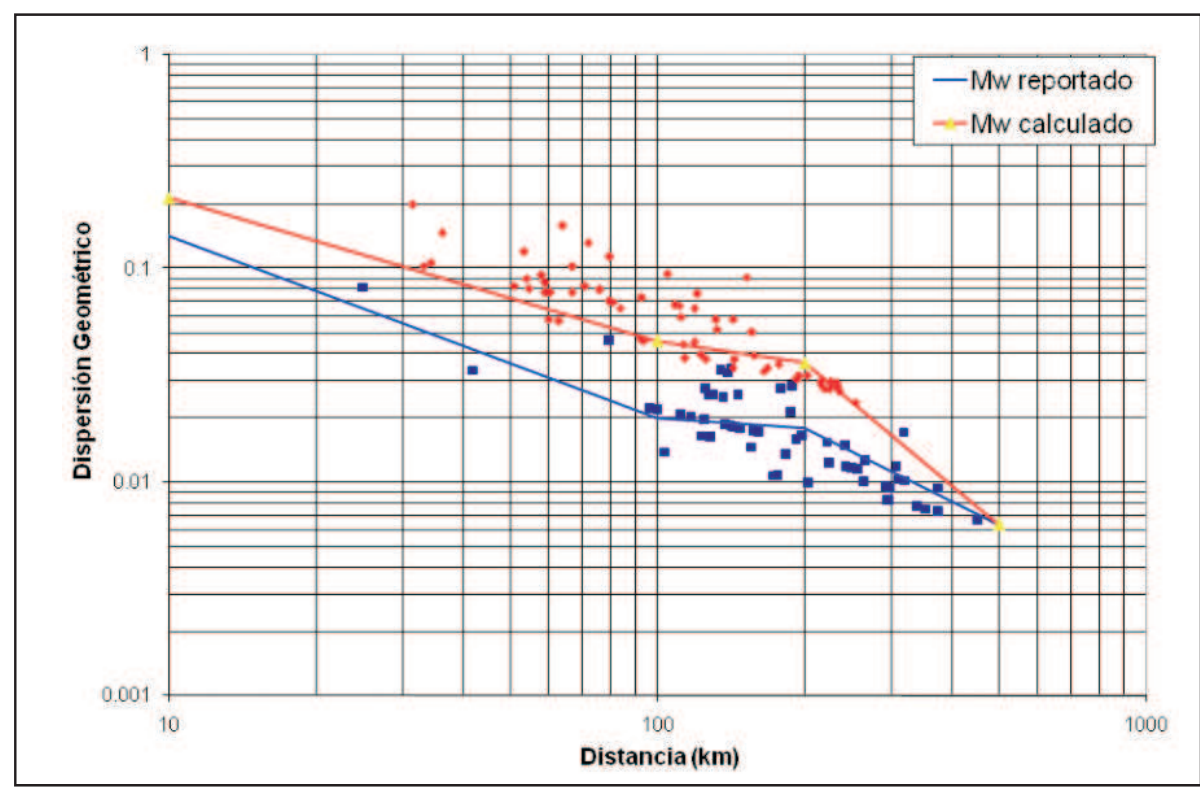

Figura 8. Dispersión geométrica de los Sismos de zona Activa para magnitudes Mw calculadas y reportadas por el NEIC.

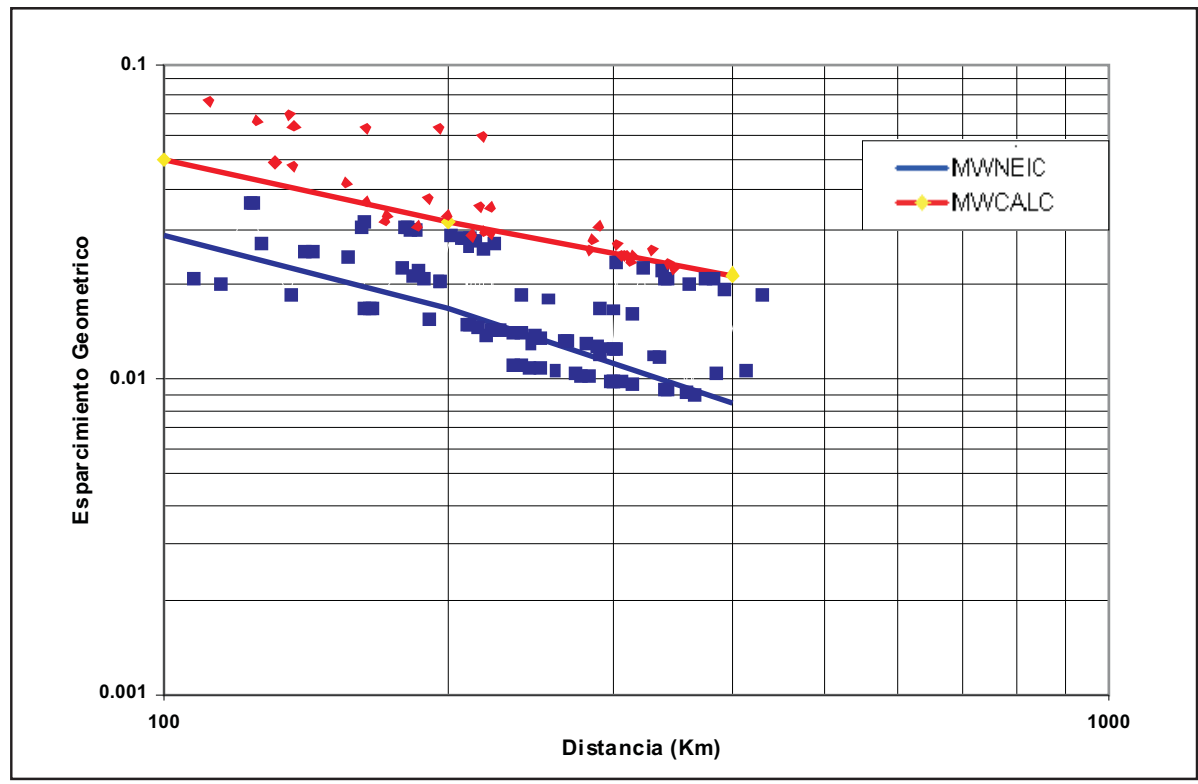

Figura 9. Dispersión geométrica de los Sismos de Subducción para magnitudes Mw calculadas y Mw reportadas por el NEIC. 


\section{Obtención de señales.}

Para este estudio los parámetros libres calibrados para las zona Activa y Zona de Subducción se presentaron en la tabla 1. Con estos datos de entrada se procedió a realizar las simulaciones para generar las señales que representan el movimiento fuerte, para las parejas $M-d$ obtenidas en Ramos (2006).

En la figura 10 se presentan las señales simuladas para las parejas M-d obtenidas en Ramos (2006) para una de las fuentes sísmicas definidas en su estudio, con sus respectivos espectros de amplitudes de Fourier, en este caso la falla Romeral.
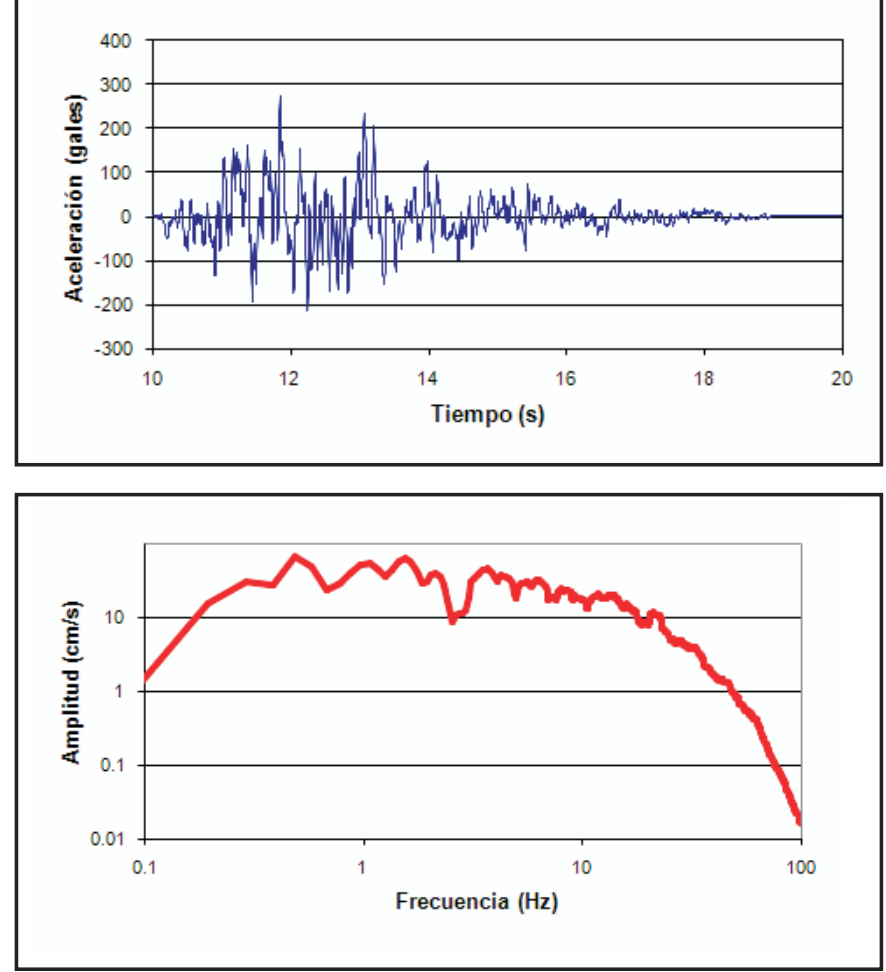

Figura 10. Señal sintética y Espectro de amplitudes de Fourier, para un sismo producido por la falla Romeral, para $M w=6.6$ y $d=54.03 \mathrm{~km}$.

\section{GENERACIÓN DE ESPECTROS DE RESPUESTA}

\section{Señales para cada perfil de suelo}

Las señales obtenidas para las parejas $M-d$ no tienen incluidos efectos locales y de sitio como aquéllos inducidos por los depósitos de suelos; es decir que las señales obtenidas por el programa SMSIM son tomadas en roca. Como el objetivo de este proyecto es el generar espectros de respuesta acordes con el estudio de microzonificación sísmica de la ciudad de Armenia, se hizo necesario incluir los efectos de los depósitos de suelo para incluir la amplificación de las señales generadas en roca. Para lograr este propósito, se utilizó el programa de modelación unidimensional de depósitos de suelo EERA, donde se simularon los perfiles de suelo con sus propiedades geotécnicas (Ingeominas, 1999).

Una vez determinadas las señales para las parejas $M-d$ en cada perfil de suelo, se procedió a generar los respectivos espectros de respuesta de desplazamiento relativo. Para obtener estos espectros se utilizó el programa SeismoSignal (Seismosoft). Es así como se obtuvieron espectros de respuesta de desplazamientos para cada perfil y seis niveles de amortiguamiento.

En las figuras 11,12 y 13 , se observan los espectros de respuesta de desplazamientos, acordes con las señales simuladas.

\section{ESPECTROS DE DISEÑO POR DESPLAZAMIENTO}

Una vez obtenido los espectros de respuesta, se obtuvieron los espectros de diseño por desplazamiento, cuya forma general suavizada son líneas rectas, tal como se aprecia en la figura 14, estos para ser usados como espectros de diseño para la metodología de diseño sísmico basado directamente en desplazamientos, para los escenarios más importantes de amenaza sísmica regional dado en Ramos (2006).

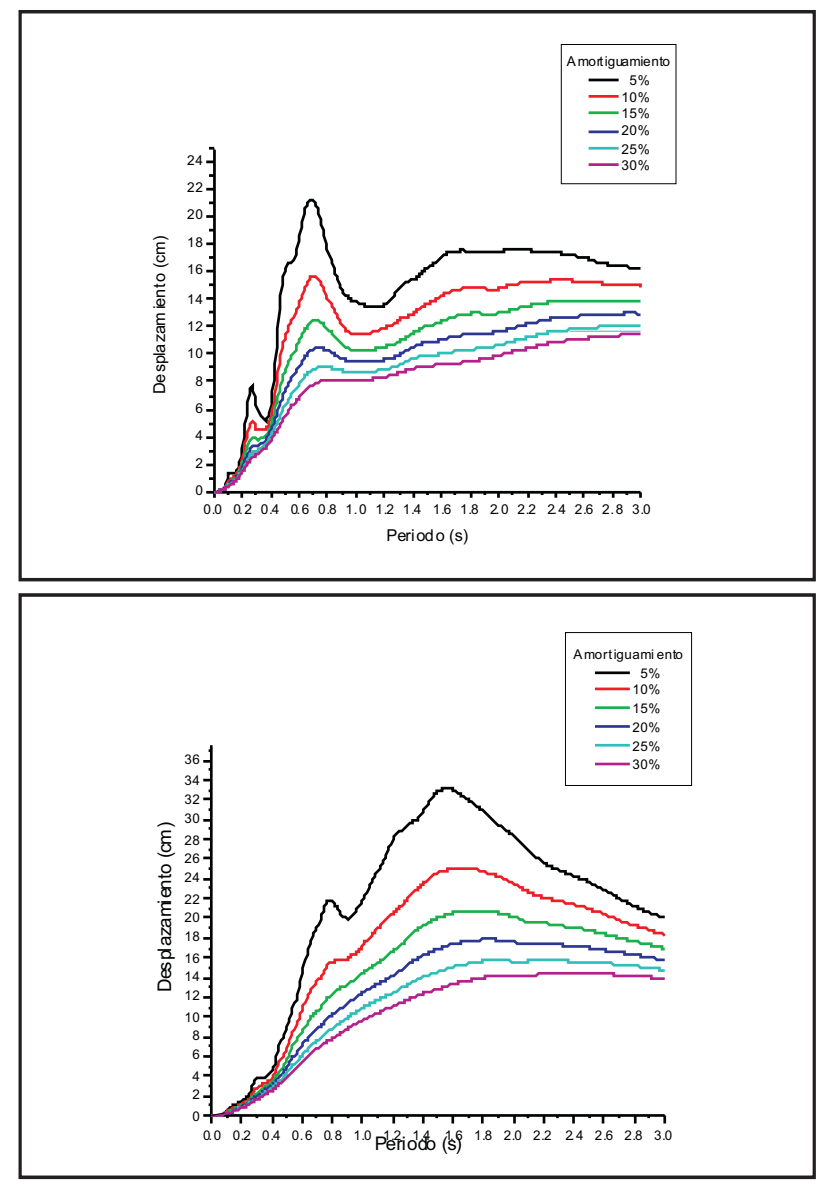




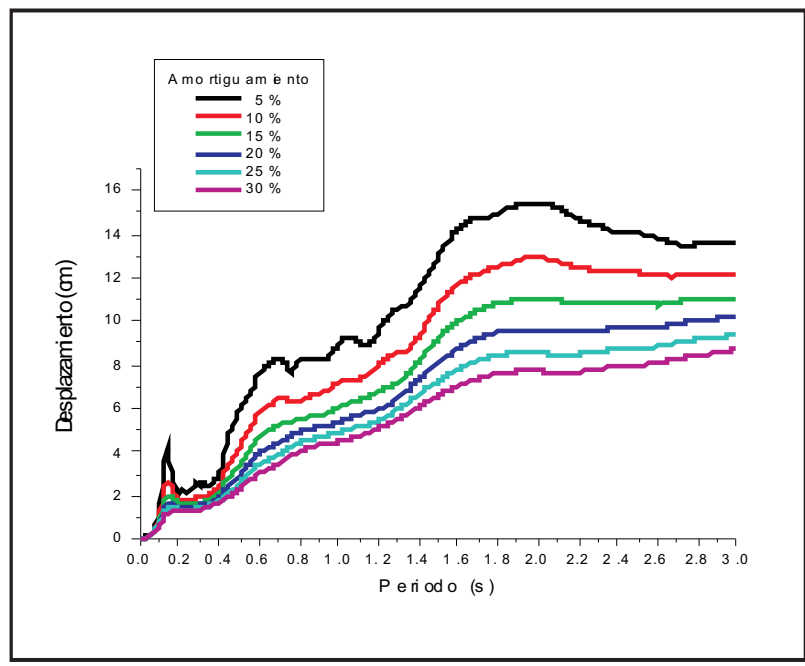

Figura 11. Espectro de Respuesta de desplazamiento relativo para los sismos de la falla Romeral, generados para la Zona $A, B, C$, respectivamente definidas en el decreto 079 de 1999.
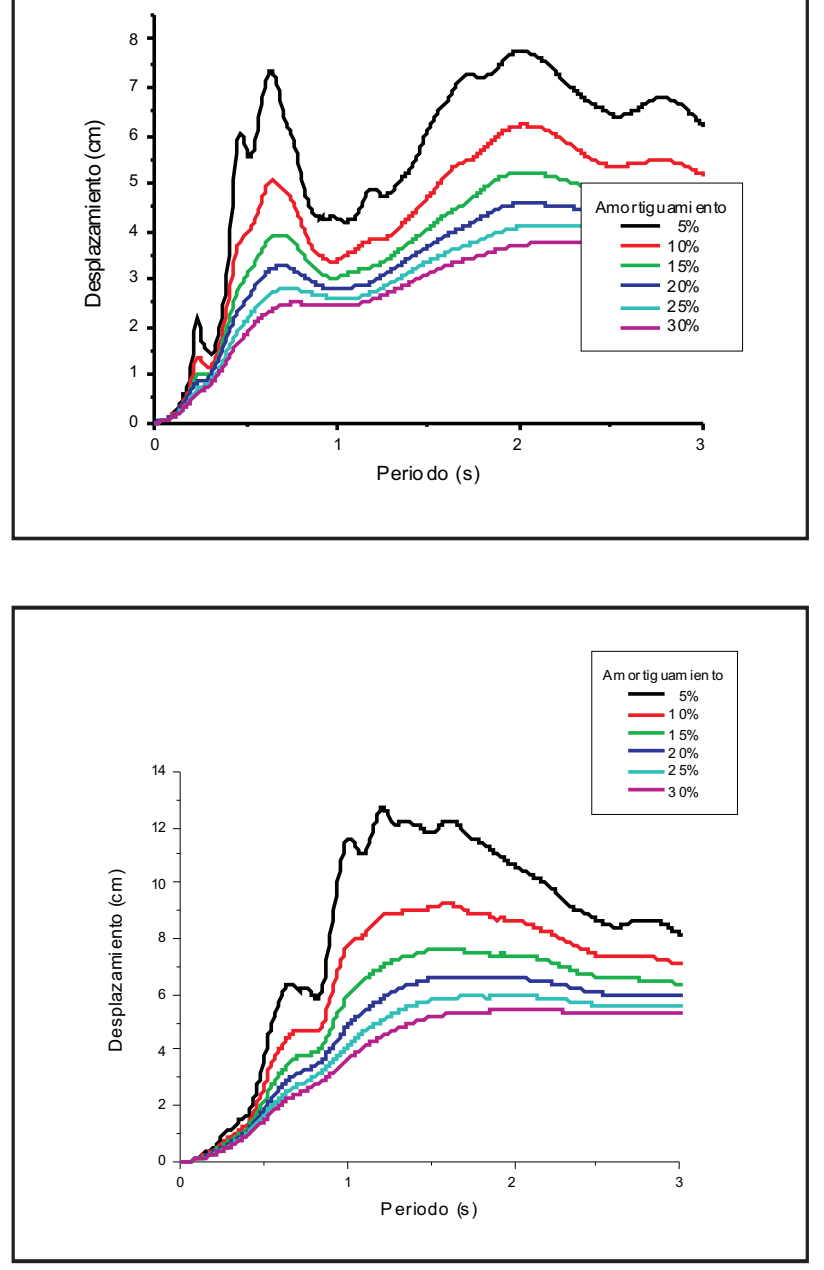

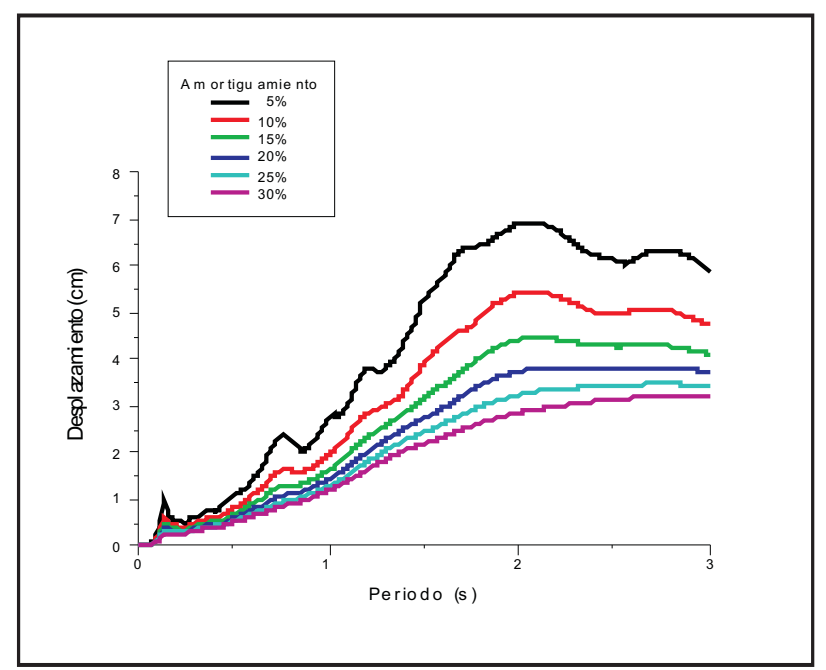

Figura 12. Espectro de Respuesta de desplazamiento relativo para los sismos de la falla lbagué, generados para la Zona $\mathrm{A}, \mathrm{B}, \mathrm{C}$, respectivamente definidas en el decreto 079 de 1999.
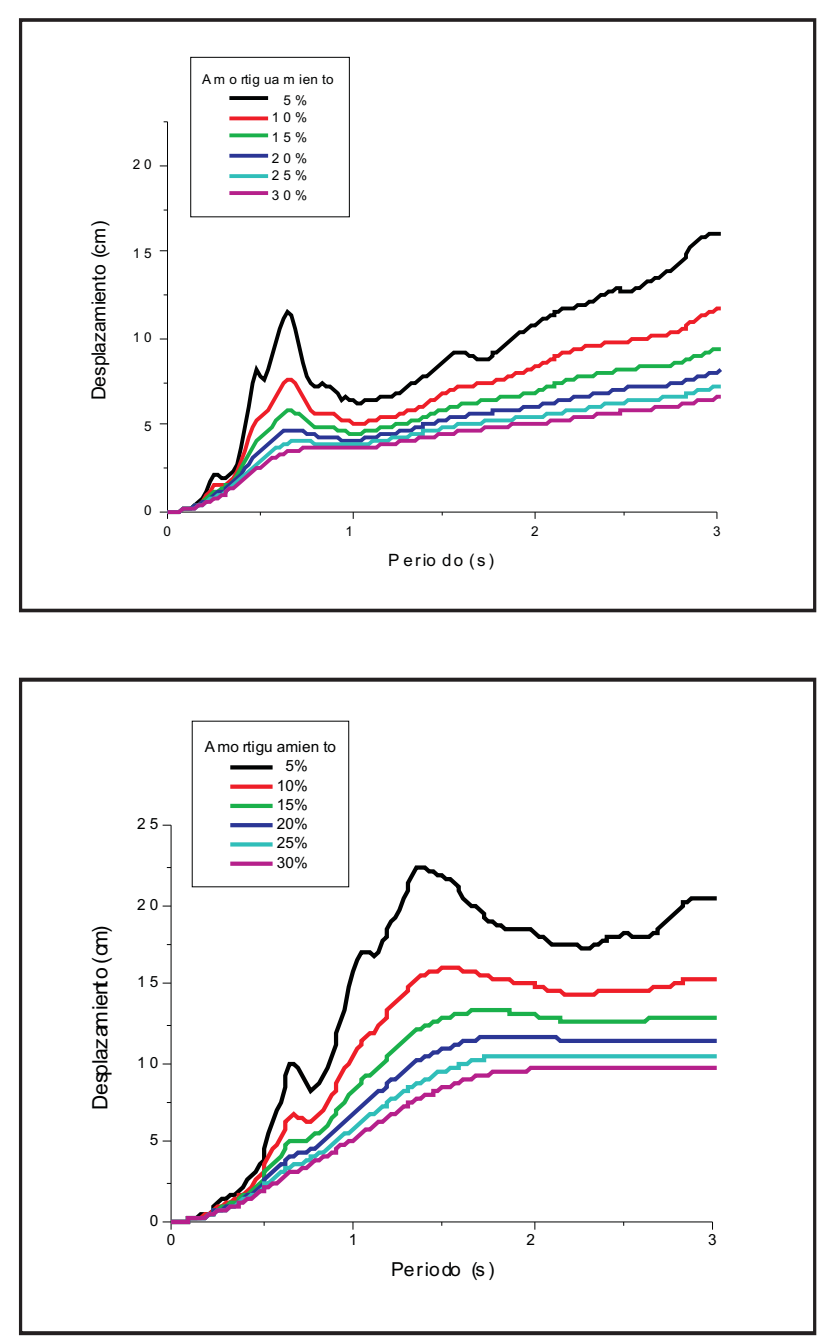


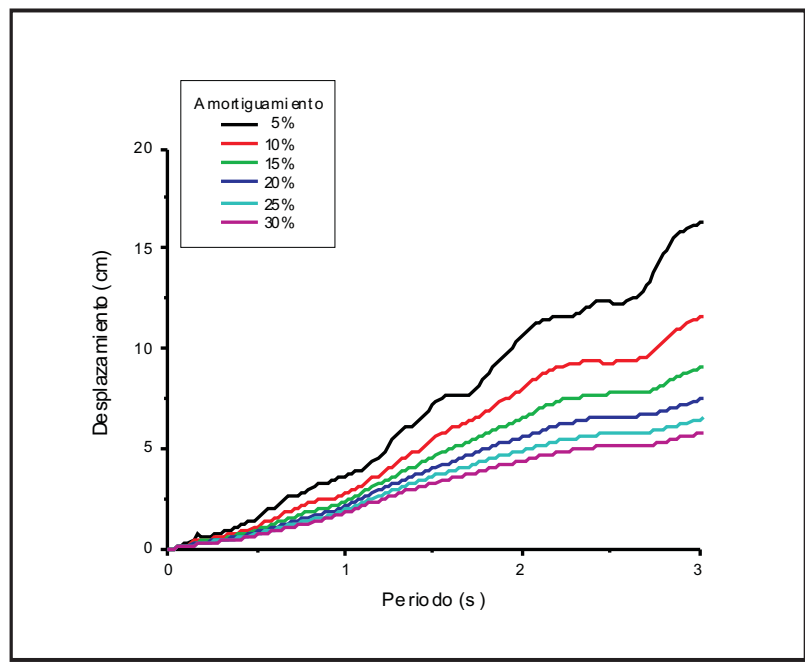

Figura 13. Espectro de Respuesta de desplazamiento relativo para los sismos de la zona de subducción, generados para la Zonas A,B y C, respectivamente, definidas en el decreto 079 de 1999.

A continuación se presentan las tablas de tiempos de control (tablas 5 a 8) y su respectivo desplazamiento según el nivel de amortiguamiento y también los gráficos de los espectros de desplazamiento de cada zona.

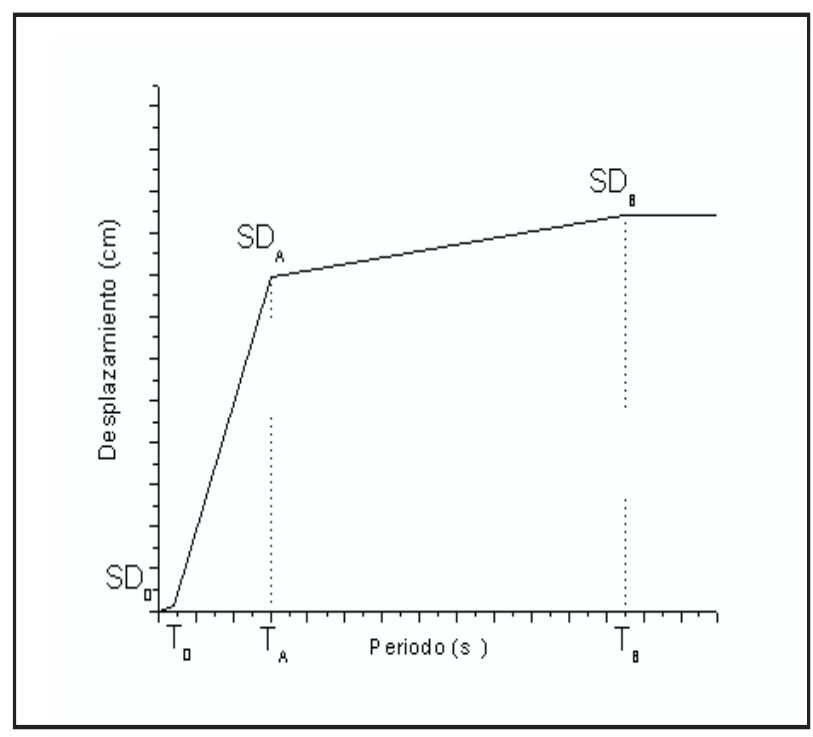

Figura 14. Linealización del espectro de diseño por desplazamiento

Tabla 5. Tiempos de control de los espectros de diseño de desplazamientos

\begin{tabular}{|c|c|c|c|c|c|c|c|c|c|}
\hline & \multicolumn{3}{|c|}{$\mathrm{T}_{0}$} & \multicolumn{3}{|c|}{$\mathrm{T}_{\mathrm{A}}$} & \multicolumn{3}{|c|}{$\mathrm{T}_{\mathrm{B}}$} \\
\hline & Zona A & Zona B & Zona C & Zona A & Zona B & Zona C & Zona A & Zona B & Zona C \\
\hline Romeral & 0.08 & 0.07 & 0.04 & 0.60 & 0.90 & 0.07 & 2.50 & 2.00 & 2.00 \\
\hline Ibagué & 0.07 & 0.09 & 0.05 & 0.6 & - & - & & 1.4 & 2 \\
\hline Subducción & 0.07 & 0.1 & 0.07 & 0.7 & - & 2.3 & - & 1.6 & 3 \\
\hline
\end{tabular}

Tabla 6. Ordenadas de control de los espectros de diseño para diferentes niveles de amortiguamiento escenario Romeral

\begin{tabular}{|c|c|c|c|c|c|c|c|c|c|c|c|c|c|c|c|c|c|c|}
\hline \multirow[b]{3}{*}{ ZONA } & \multicolumn{18}{|c|}{ AMORTIGUAMIENTO } \\
\hline & \multicolumn{3}{|c|}{$5 \%$} & \multicolumn{3}{|c|}{$10 \%$} & \multicolumn{3}{|c|}{$15 \%$} & \multicolumn{3}{|c|}{$20 \%$} & \multicolumn{3}{|c|}{$25 \%$} & \multicolumn{3}{|c|}{$30 \%$} \\
\hline & $\mathrm{SD}_{0}$ & $\mathrm{SD}_{\mathrm{A}}$ & $\mathrm{SD}_{\mathrm{B}}$ & $\mathrm{SD}_{0}$ & $\mathrm{SD}_{\mathrm{A}}$ & $\mathrm{SD}_{\mathrm{B}}$ & $\mathrm{SD}_{0}$ & $\mathrm{SD}_{\mathrm{A}}$ & $\mathrm{SD}_{\mathrm{B}}$ & $\mathrm{SD}_{0}$ & $\mathrm{SD}_{\mathrm{A}}$ & $\mathrm{SD}_{\mathrm{B}}$ & $\mathrm{SD}_{0}$ & $\mathrm{SD}_{\mathrm{A}}$ & $\mathrm{SD}_{\text {в }}$ & $\mathrm{SD}_{0}$ & $\mathrm{SD}_{\mathrm{A}}$ & $\mathrm{SD}_{\text {в }}$ \\
\hline A & 0.2 & 15.9 & 18.8 & 0.2 & 13.2 & 16.0 & 0.2 & 11.3 & 14.2 & 0.2 & 9.7 & 12.9 & 0.2 & 8.4 & 11.7 & 0.2 & 7.6 & 10.7 \\
\hline B & 0.1 & 26.8 & 33.0 & 0.1 & 20.9 & 27.2 & 0.1 & 17.0 & 22.0 & 0.1 & 14.0 & 18.5 & 0.1 & 12.2 & 16.0 & 0.1 & 10.8 & 14.5 \\
\hline C & 0.0 & 8.5 & 14.8 & 0.0 & 7.0 & 12.4 & 0.0 & 5.8 & 10.8 & 0.0 & 5.0 & 9.5 & 0.0 & 4.2 & 8.5 & 0.0 & 3.7 & 7.8 \\
\hline
\end{tabular}


Tabla7. Ordenadas de control de los espectros de diseño para diferentes niveles de amortiguamiento escenario Ibagué

\begin{tabular}{|c|c|c|c|c|c|c|c|c|c|c|c|c|c|c|c|c|c|c|}
\hline \multirow[b]{3}{*}{ ZONA } & \multicolumn{18}{|c|}{ AMORTIGUAMIENTO } \\
\hline & \multicolumn{3}{|c|}{$5 \%$} & \multicolumn{3}{|c|}{$10 \%$} & \multicolumn{3}{|c|}{$15 \%$} & \multicolumn{3}{|c|}{$20 \%$} & \multicolumn{3}{|c|}{$25 \%$} & \multicolumn{3}{|c|}{$30 \%$} \\
\hline & $\mathrm{SD}_{0}$ & $\mathrm{SD}_{\mathrm{A}}$ & $\mathrm{SD}_{\mathrm{B}}$ & $\mathrm{SD}_{0}$ & $\mathrm{SD}_{\mathrm{A}}$ & $\mathrm{SD}_{\mathrm{B}}$ & $\mathrm{SD}_{0}$ & $\mathrm{SD}_{\mathrm{A}}$ & $\mathrm{SD}_{\text {в }}$ & $\mathrm{SD}_{0}$ & $\mathrm{SD}_{\mathrm{A}}$ & $\mathrm{SD}_{\text {в }}$ & $\mathrm{SD}_{0}$ & $\mathrm{SD}_{\mathrm{A}}$ & $\mathrm{SD}_{\text {в }}$ & $\mathrm{SD}_{0}$ & $\mathrm{SD}_{\mathrm{A}}$ & $\mathrm{SD}_{\text {в }}$ \\
\hline$A$ & 0.0 & 5.7 & 7.0 & 0.0 & 4.6 & 6.0 & 0.0 & 3.7 & 5.2 & 0.0 & 3.1 & 4.6 & 0.0 & 2.6 & 4.1 & 0.0 & 2.3 & 3.8 \\
\hline B & 0.0 & - & 12.5 & 0.0 & - & 9.8 & 0.0 & - & 7.7 & 0.0 & - & 6.4 & 0.0 & - & 5.7 & 0.0 & - & 5.3 \\
\hline C & 0.0 & - & 7.0 & 0.0 & - & 5.4 & 0.0 & - & 4.5 & 0.0 & - & 3.9 & 0.0 & - & 3.4 & 0.0 & - & 3.0 \\
\hline
\end{tabular}

Tabla 8.: Ordenadas de control de los espectros de diseño para diferentes niveles de amortiguamiento escenario Subducción

\begin{tabular}{|c|c|c|c|c|c|c|c|c|c|c|c|c|c|c|c|c|c|c|}
\hline \multirow[b]{3}{*}{ ZONA } & \multicolumn{18}{|c|}{ AMORTIGUAMIENTO } \\
\hline & \multicolumn{3}{|c|}{$5 \%$} & \multicolumn{3}{|c|}{$10 \%$} & \multicolumn{3}{|c|}{$15 \%$} & \multicolumn{3}{|c|}{$20 \%$} & \multicolumn{3}{|c|}{$25 \%$} & \multicolumn{3}{|c|}{$30 \%$} \\
\hline & $\mathrm{SD}_{0}$ & $\mathrm{SD}_{\mathrm{A}}$ & $\mathrm{SD}_{\text {в }}$ & $\mathrm{SD}_{0}$ & $\mathrm{SD}_{\mathrm{A}}$ & $\mathrm{SD}_{\mathrm{B}}$ & $S D_{0}$ & $\mathrm{SD}_{\mathrm{A}}$ & $\mathrm{SD}_{\mathrm{B}}$ & $\mathrm{SD}_{0}$ & $\mathrm{SD}_{\mathrm{A}}$ & $\mathrm{SD}_{\text {в }}$ & $\mathrm{SD}_{0}$ & $\mathrm{SD}_{\mathrm{A}}$ & $\mathrm{SD}_{\mathrm{B}}$ & $\mathrm{SD}_{0}$ & $\mathrm{SD}_{\mathrm{A}}$ & $\mathrm{SD}_{\mathrm{B}}$ \\
\hline A & 0.0 & 10.5 & - & 0.0 & 7.7 & - & 0.0 & 6.0 & - & 0.0 & 4.7 & - & 0.0 & 2.6 & 4.1 & 0.0 & 3.6 & - \\
\hline B & 0.0 & - & 22.0 & 0.0 & - & 16.4 & 0.0 & - & 13.8 & 0.0 & - & 12.0 & 0.0 & - & - & 0.0 & - & 9.7 \\
\hline C & 0.0 & 12.0 & 13.0 & 0.0 & 9.5 & 10.4 & 0.0 & 7.8 & 8.6 & 0.0 & 6.7 & 7.4 & 0.0 & - & 5.8 & 0.0 & 5.2 & 5.8 \\
\hline
\end{tabular}

DISCUSIÓN

Si se hace una comparación de los resultados de los EAF obtenidos en este estudio y los resultados obtenidos en otras investigaciones, se puede notar algunas diferencias, sobre todo con los factores de calidad, pero un cierto grado de aproximación con los demás factores, la diferencia puede radicar en la diferencia de sismos utilizados en cada estudio y la forma en que estos fueron procesados, previo a ejecutar la regresión no lineal y de esta manera calcular los parámetros libres $R_{\text {甲巾 }}, Q_{o}, Q_{l}, \kappa_{l}, \varepsilon$ antes mencionados.

Otra situación encontrada en este estudio es el uso generalizado de la magnitud $\mathrm{Ml}$, en primer lugar porque se satura por debajo de $\mathrm{Ml}=6.5$, además que las correlaciones con Mw y Ms, tienen demasiada dispersión y son válidas para $4.5<\mathrm{Ml}$, por lo tanto el procedimiento más adecuado en tomarlas de los reportes de catálogos internacionales, por ejemplo NEIC, Harvard, los cuales reportan la magnitud Mw de una inversión del tensor de momento.

\section{SIMULACIÓN DE SEÑALES}

Las señales sintéticas generadas utilizando la teoría del espectro radiado y la técnica de simulación estocástica de movimiento fuerte utilizada en este estudio, representan la radiación de la alta frecuencia, describiendo así la distribución de amplitudes y frecuencias predominantes para el sismo en cuestión. Debido a que la señal parte de un ruido numérico, conocido como ruido Gaussiano, la metodología no permite conocer la forma de onda de la señal en particular. Además, los sismos generados con el procedimiento utilizado en este estudio, no son sismos para una falla en particular, dado que el modelo del espectro de fuente al asumir el principio de similaridad para poder presentar una ley de escalamiento entre magnitudes, es a la vez, un modelo de parámetro de esfuerzo constante; lo que indica que los sismos que se simularon para la región en estudio, tienen un parámetro de esfuerzos constante promedio para la zona de fallas corticales y subducción respectivamente (nótese que se habla de dos escenarios tectónicos, más no de una falla en particular). De igual manera, los parámetros que se calibraron en los modelos EAF de falla puntual y finita, son representativos de cada región sísmica y se obtuvieron a partir de regresiones de la información sísmica registrada en la zona. Hay modelos más refinados para simular señales para una falla en particular, donde se requiere un estudio muy detallado de las funciones de deslizamiento, de las asperidades y de los mecanismos focales de los sismos que se han generado es esa falla en particular (Aki , 1967). Estos modelos se basan en el estudio de la cinemática del frente de ruptura y aunque son más precisos (métodos determinista), en nuestro medio no se cuenta con estudios tan detallados de cada una de las fallas que compone los megafallamientos de 
nuestro país. Por ello las alternativas probabilísticas como el método utilizado en este estudio, son apropiadas desde la perspectiva ingenieril para representar la sismicidad promedio de la región para las fuentes de amenaza descritas en Ramos (2006). Las señales simuladas sólo representan el movimiento fuerte contenido dentro del paquete de ondas de corte $S$ y para distancias grandes en las ondas superficiales; por ello la radiación de altas frecuencias se presenta, pero las señales obtenidas es este estudio carecen por su misma naturaleza de radiación de bajas frecuencias, lo cual se vio reflejado en los espectros de desplazamiento, en donde a partir de un período de 3 segundos, las señales obtenidas no generaban ordenadas espectrales de desplazamiento confiables, debido a la sensibilidad de los desplazamientos respecto a la radiación de la baja frecuencia. Caso contrario sucede con las ordenadas de aceleración, que son muy dependientes de la radiación de la alta frecuencia. Por ello se vio una buena correlación de las aceleraciones máximas netas registradas con las simuladas y un buen desempeño frente a ecuaciones de atenuación y estudios previos como el de Gallego (2000), donde se utilizó la metodología de simulaciones estocásticas, pero generando las aceleraciones máximas probables mediante teoría de vibraciones aleatorias.

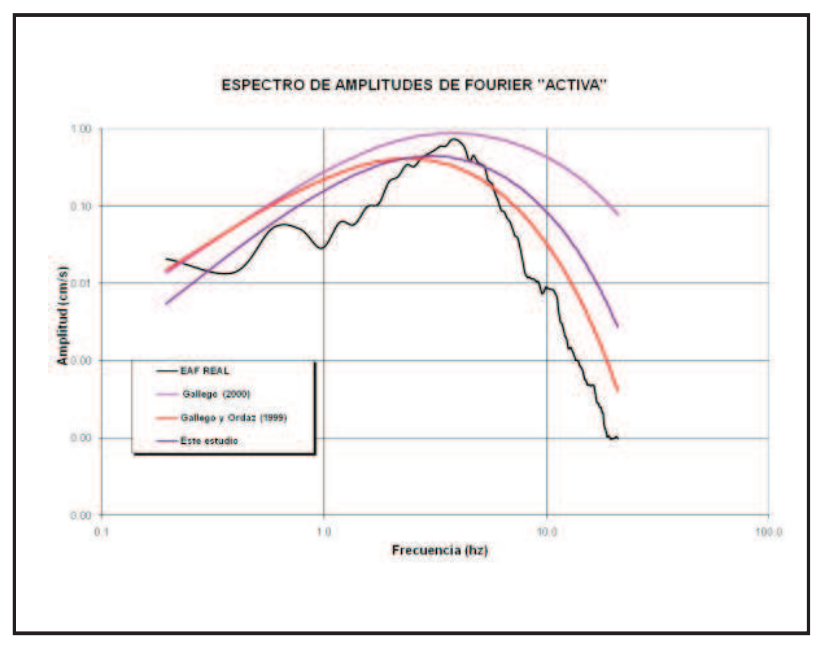

Figura 15. Comparación de los espectros de amplitudes de Fourier (EAF) de diferentes estudios

\section{FUNCIÓN DE DURACIÓN Y DISPERSIÓN GEOMÉTRICA}

Para la aplicación de la metodología utilizada en este estudio, era necesario construir las funciones de tiempo y esparcimiento geométrico, para expresar de forma paramétrica la duración de los sismos en función de la distancia y la atenuación dependiente de la distancia e independiente de la frecuencia de las ondas sísmicas respectivamente. La mayor dificultad para determinar estas curvas es la falta de información y calidad de los registros acelerográficos analizados.

La curva de duración presentó un comportamiento trilineal, similar a las obtenidas en Atkinson y Boore (1995). Para el caso analizado en este estudio, se encontró que la distancia para la cual las ondas de cuerpo dejan de predominar el frente de onda es para $100 \mathrm{~km}$, presentando un aumento de la duración con la distancia. En el rango de distancia que está entre $100 \mathrm{~km}$ y $200 \mathrm{~km}$, las ondas presentan una transición, donde se refleja las reflexiones de las ondas en la discontinuidad de Moho, presentando un incremento muy pequeño de la duración con respecto a la distancia. De 200 $\mathrm{km}$ en adelante, en el movimiento predominan las ondas superficiales (Lg) y la duración aumenta con la distancia. La función para modelar la dispersión geométrica, se determinó utilizando un modelo bilineal para realizar un primer ajuste de los parámetros libres del EAF; Luego se realizó un ajuste del modelo de dispersión geométrica, representándolo así como un modelo trilineal. Éste modelo se ajustó sólo para los sismos cuya magnitud de momento sísmico (Mw) estaba reportado en las agencias internacionales, para así poder determinar los valores de los parámetros, para ingresar como dato de entrada al programa SMSIM V2.0 (Boore, 2003). EI modelo se ajustó de acuerdo a los parámetros establecidos y para las condiciones de la región en estudio.

Las curvas de dispersión y duración se generaron, pero deben ser estudios más detallados para nuestra región, de modo que se obtengan unas funciones de atenuación geométrica y tiempo más exactas.

\section{OBTENCIÓN DE ESPECTROS DE RESPUESTA DE DESPLAZAMIENTO}

Las señales simuladas para las fuentes de amenaza sísmica antes mencionadas, fueron filtradas a través de los depósitos de suelo definidos en el estudio de Ingeominas (1999) utilizando el programa SHAKE (versión bajo excell, EERA 2000). Se observó que las señales filtradas a través de los depósitos de suelo generaron espectros de desplazamiento de diferente tipología para cada perfil. Por ejemplo, los espectros de respuesta obtenidos en las zonas de cenizas, presentaron una tendencia de tres pendientes en su desarrollo y los desplazamientos mayores se dieron a períodos de $0.3 \mathrm{~s}$ y $0.8 \mathrm{~s}$, donde se ve un claro acercamiento al acoplamiento entre las frecuencias de la señal con la frecuencia natural del depósito de suelo. Para un nivel de amortiguamiento del 30\%, dicha amplificación se atenúa por efectos de este valor. Para la ciudad de Armenia se realizó un estudio para la evaluación de efectos de sitio, en donde se encontraron unos períodos de respuesta de los depósitos de 
suelo que oscilaban entre 0.36 y 0.59 segundos (Bermúdez, et al. 2000). Otra característica que se encontró es que para los depósitos de cenizas, las amplificaciones de la aceleración fueron mayores que para el perfil de suelo denominado llenos; esto explica que las cenizas tienden a ser más rígidas que los llenos, lo cual también se evidenció en los espectros de desplazamiento, donde los mayores valores se dieron en el perfil llenos, lo cual hace ver que un suelo con características rígidas, presenta mayores aceleraciones y menores desplazamientos; caso contrario se ve para suelos blandos.

Para determinar hasta que períodos eran confiables las ordenadas espectrales, se tomo una señal simulada para una magnitud $\mathrm{Mw}=6.5$ y distancia hipocentral de $21.41 \mathrm{~km}$ pasada por el perfil de suelo cenizas 1 , de la microzonificación de Armenia. A esta señal se le eliminaron las bajas frecuencias utilizando diferentes filtros, en donde para cada filtrado se generaron los espectros de respuesta de desplazamiento; este ejercicio se puede ver en la figura 16.

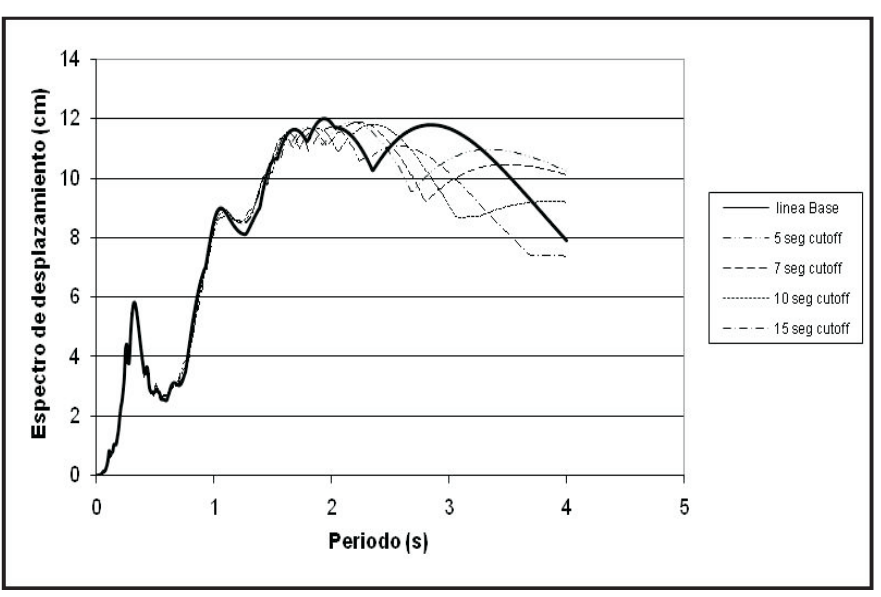

Figura 16. Determinación de la sensibilidad de los desplazamientos, respecto a diferentes filtros que cortan las bajas frecuencias para un sismo de $M w=6.5$ y $D=21.41$ km generado sintéticamente y pasado a través del perfil de suelo Cenizas 1, de la microzonificación de Armenia. Nótese las desviaciones de las ordenadas espectrales cerca de 3 segundos.

Como se evidencia en la figura 16, las ordenadas espectrales para las señales sintéticas, son pobres en la radiación de bajas frecuencias, por lo cual se decidió presentar los espectros hasta un período de 3 segundos.

Más allá de este valor, las ordenadas espectrales que se presenten no son confiables. Claro está que para 3 segundos, ya es evidente la caída del desplazamiento, que corresponde a la baja frecuencia, pero dado que el diseño basado directamente en desplazamientos necesita ordenadas para periodos altos, se decidió dejar los espectros hasta 3 segundos. Debido a la incertidumbre de las ordenadas predichas a partir de 3 segundos, las amplitudes de los desplazamientos se toman constantes, tal y como se presentan en los espectros de diseño presentados en el anexo $\mathrm{H}$.

\section{CONCLUSIONES}

Se encontró que los parámetros libres del espectro de amplitudes de Fourier, calibrados mediante regresiones no lineales, aunque variaron en comparación con otros estudios, predicen movimientos del terreno que son acordes con los sismos observados.

Se observó que las señales simuladas en este estudio, representan la radiación de la alta frecuencia y su distribución en el tiempo; más no especifican ningún tipo de forma de onda, ni representan un frente de ruptura en particular y tampoco tienen en cuenta efectos locales. Por ello son apropiadas para fines ingenieriles donde se necesite realizar análisis cronológico para estructuras complejas y especiales.

Los espectros de respuesta de desplazamiento obtenidos, presentaron unos picos para el rango de periodos entre $0.3 \mathrm{y}$ 0.8 segundos, debido a la resonancia presentada entre el contenido frecuencial de las señales y los períodos de vibración natural de los depósitos de suelo.

Se encontró que las ordenadas de desplazamiento obtenidas, presentan mayores valores de amplitud espectral para los depósitos de suelo blando que para depósitos rígidos.

Debido a que los desplazamientos del terreno son muy sensibles a la radiación de la baja frecuencia y las señales simuladas son pobres en esta, se determinó que las ordenadas espectrales de desplazamiento obtenidas en este estudio son confiables si se generan hasta un periodo de $3 \mathrm{~s}$. Para periodos mayores se toma como constante las ordenadas de desplazamiento. Queda así abierta la discusión para buscar modelos de fuente más precisos que permitan simular señales con una buena radiación de baja frecuencia, para que los espectros de desplazamiento puedan ser llevados más allá de los $3 \mathrm{~s}$.

Los valores de aceleración máxima determinados en este estudio, comparadas con las aceleraciones predichas por las leyes de atenuación utilizadas en Colombia, fueron menores; lo que concluye que dichas leyes de atenuación sobrestiman los valores de aceleración máxima.

Se generaron los espectros de repuesta de seudoaceleración y al ser comparados con el espectro de diseño propuesto 
para la ciudad de Armenia, se observó que las amplitudes espectrales de aceleración son mayores y se destacó un acortamiento en los períodos de control de la zona plana para el espectro generado para el perfil de cenizas 1 y 2 . Para los perfiles Cenizas 3 y llenos se presentaron unos picos que van más allá de la zona plana del espectro. Por lo cual, debe entrar a discusión el valor del período de control para la zona plana de los espectros de diseño establecidos para la ciudad de Armenia.
En este estudio se presentó como aporte adicional los espectros de diseño para cada fuente de amenaza sísmica, apropiados para el diseño sísmico basado directamente en desplazamientos. Las amplitudes espectrales obtenidas son coherentes y al ser comparadas con ambientes tectónicos con condiciones locales semejantes a la nuestra, se concluye que los valores obtenidos están dentro de márgenes aceptables.

\section{AGRADECIMIENTOS}

A INGEOMINAS, en especial a la Red Nacional de Acelerografos de Colombia (RNAC) por el suministro de las trazas acelerográficas desde junio del 1993 hasta mayo del 2005 que fueron usadas en este proyecto, al Observatorio Sismológico de la Universidad del Quindío y su personal, en especial, a la Ingeniera Marisol Gómez Cano y a la Geóloga Isabel Cristina Correa, a la Universidad del Quindío y la Facultad de Ingeniería.

\section{BIBLIOGRAFÍA}

Aki. K. (1967). Scaling law of seismic spectrum, J. Geophys. Res. 72, 1217-1231.

Anderson, J.G. and S.E. Hough (1984). A model for the shape of the Fourier amplitude spectrum of acceleration at high frequencies, Bull. Seism. Soc. Am. 74, 1969-1993.

Atkinson, G.M. and R.F. Mereu (1992). The shape of ground motion attenuation curves in southeastern Canada, Bull. Seism. Soc. Am. 82, 2014-2031.

Atkinson, G. M. (1993a), Notes on Ground Motion Parameters for Eastern North America: Duration and H/V Ratio, Bull. Seismol. Soc. Am. 83, 587-596.

Atkinson, G. M. and Boore, D. M. (1995), Ground Motion Relations for Eastern North America, Bull. Seismol. Soc. Am. 85, 17-30.

Bermudez-Angulo, M.L., Monsalve-Jaramillo, H., Cuenca, J.C, Vargas-Jimenez, C.A, Chavez-García, F., Ordaz, M. (2000). Evaluación de efectos de sitio en la ciudad de Armenia, colombia. Instituto Panamericano de Geografía e Historia- IPGH, México, Proyecto Geof. 3.4.2.45.

Bommer, J. J, Elnashai A. S.(1999), “Displacement Design Spectra for Seismic Design”, Journal of Earthquake Enginneering, Vol. 3, No. 1,1-32.

Boore. D. M. (1983). Stochastic simulation of high-frecuency ground motion based on seismological models of radiated spectra, Bull. Seism. Soc. Am. 73,1865-1884.

Boore, D.M., 2000, SMSIM-Fortran Programs for Simulating Ground Motions From Earthquakes:Version 2.0 - A Revision of OFR 96-80-A, U.S. Geological Survey Open File Report OFR 00-509.

Boore. D. M. (2003). Simulation of ground motion using the stochastic method, pure and applied geophysics, Vol 160 (2003), 635-676.

Brune, J. N. (1970). Tectonic Stress and the spectra of seismic S waves from earth, J. Geophys. Res. 75, 4997- 5009.

Burguer, R., P. Somerville, J. Barker, R Herrmann, and D. Helmberger (1987). The effect of crustal structure on strong ground motion attenuation relations in eastern North America, Bull. Seism. Soc. Am. 77, 420-439.

Cartwright, D. E. and Longuet-Higgins, M. S. (1956), The Statistical Distribution of the Maxima of a Random Function, Proc. R. Soc. London 237, 212-232.

Decreto 079 (1999), Decreto 079 por el cual se adopta la microzonificación sísmica y se reglamentan las secciones a2.4 y a2.6 del decreto 33 de 1998 - NSR-98, para las zonas determinadas en el mapa denominado "Zonificación Sísmica de la Ciudad de Armenia". Microzonificación Sísmica de la Ciudad de Armenia.

Federal Emergency Management Agency. NEHRP (1997) "Guidelines for the Seismic Rehabilitation of Buildings". FEMA 274. Preparado por el Applied Technology Council.

Gallego, M. y Ordaz, M. (1999), “Construcción de leyes de atenuación para la República de Colombia a partir de espectros fuentes y teoría de vibraciones aleatorias", Ingenieria Estructural, ed. Alex Barbat, Barcelona España. 
Gallego M. S. (2000), “Estimación de Riesgo Sísmico en la República de Colombia”; Tesis para obtener el grado de Maestro en Ingeniería; Universidad Nacional Autónoma de México; México D.

Gautschi, W y Cahill, W, F (1965), Exponential integral and related functions, Handbook of mathematical functions, M Abramowitz and I, Stegun, Dover publication, New york.

Hanks, T.C. (1982). fmax, Bull. Seism. Soc. Am. 72, 1867-1879.

Haskell, N.A., (1966), Total energy and energy spectral density of elastic wave radiation from propagating faults. II. A stochastic fault model. Bull. Seism. Soc. Am., 56, 125-140.

Ingeominas, (1999). Terremoto del Quindio (Enero 25 de 1999), Informe Técnico-Científico. Volumen II: Zonificación Sismogeotécnica Indicativa Para la Reconstrucción de Armenia. INGEOMINAS.

Ingeominas, 1993. Modelo de velocidades para el territorio colombiano. En: Red Sismológica Nacional de Colombia. Bogotá, inédito.

Kanamori, H., 1977, The energy release in great earthquakes, J. Geophys. Res. 82, 2981-2987.

Kowalsky, M. J. and Priestley, M. N. J.(2000) “Direct Displacement Based Seismic Design of Concrete Buildings”, Bull. of NZSEE, Vol. 33, No.4, pp 403-420.

Monsalve, H., (1998), Geometría de la Subducción de la Placa Nazca en el Noroeste de Colombia: Implicaciones tectónicas y sísmicas, Tesis de Maestría, Universidad Nacional Autónoma de México.

Monsalve, H., (2005). Solución matemática a la integral exponencial de Cahill, comunicada personalmente. No ha sido publicada oficialmente.

Ojeda, A. E. Y Martinez S. E. (1998), "Modelo para la atenuación de la energía sísmica en Colombia a partir de sismos registrados por la red nacional de acelerografos", XII Jornadas Estructurales de la Ingeniería de Colombia. Santa Fe de Bogotá.

Ordaz, M. y Singh, S.K. (1992), Source spectra and spectral attenuation of seismic waves from Mexican earthquakes, and evidence of amplification in the hill zone of Mexico City, Bull. Seism. Soc. Am.,82, 24-43.

Ramos, T. (2006) "Selección de señales sísmicas para microzonificación de ciudades. Caso: Armenia". Tesis para obtener el grado de ingeniero civil. Universidad del Quindío.

Restrepo, J.C; (2003), "Diseño Sísmico Basado en Desplazamientos Para Estructuras de Concreto Reforzado". Tesis para obtener el grado de ingeniero civil. Universidad del Quindío.

SEAOC(1995) "Vision 2000" - "Performance Based Seismic Engineering of Buildings" \}Sacramento California. Structural Engineers Association of California.

Singh, S K, Ordaz, M Anderson J G. Rodriguez M Quaas, R, Mena, E Ottaviani, M y Almora, D. (1989) "Análisis of near source strong ground motion recording along the Mexican Subdction zone", Bull. Seism. Soc. Am., 79, 1697-1717.

Sullivan, T.J. (2002) "The current limitations of displacement based design" MSc Dissertation, European School of Advanced Studies in Reduction of Seismic Risk (ROSE School), University of Pavia, Italy. 